\title{
Homeostatic depression shows heightened sensitivity to synaptic calcium
}

1 Catherine J. Yeates ${ }^{1,2,4}$, C. Andrew Frank ${ }^{1,2,3}$

$2{ }^{1}$ Department of Anatomy and Cell Biology, University of Iowa Carver College of Medicine, Iowa

3 City, IA, USA

$4 \quad 2$ Interdisciplinary Graduate Program in Neuroscience, University of Iowa, Iowa City, IA, USA

$5 \quad{ }^{3}$ Iowa Neuroscience Institute, University of Iowa Carver College of Medicine, Iowa City, IA, USA

$6 \quad{ }^{4}$ Present Address: Department of Biology, University of Dayton, Dayton, OH, USA

7 * Correspondence:

8 C. Andrew Frank

9 andy-frank@uiowa.edu

10 Keywords: synapse, homeostasis, depression, Drosophila melanogaster, NMJ,

11 neurotransmission, plasticity 


\section{Abstract}

Synapses and circuits rely on homeostatic forms of regulation in order to transmit meaningful information. The Drosophila melanogaster neuromuscular junction (NMJ) is a well-studied synapse that shows robust homeostatic control of function. Most prior studies of homeostatic plasticity at the NMJ have centered on presynaptic homeostatic potentiation (PHP). PHP happens when postsynaptic muscle neurotransmitter receptors are impaired, triggering retrograde signaling that causes an increase in presynaptic neurotransmitter release. As a result, normal levels of evoked excitation are maintained. The counterpart to PHP at the NMJ is presynaptic homeostatic depression (PHD). Overexpression of the Drosophila vesicular glutamate transporter (VGlut) causes an increase in the amplitude of spontaneous events. PHD happens when the synapse responds to the challenge by decreasing quantal content during evoked neurotransmission - again, resulting in normal levels of postsynaptic excitation.

We hypothesized that there may exist a class of molecules that affects both PHP and PHD. Impairment of any such molecule could hurt a synapse's ability to respond to any significant homeostatic challenge. We conducted an electrophysiology-based screen for blocks of PHD. We did not observe a block of PHD in the genetic conditions screened, but we did find loss-of-function conditions that led to a substantial deficit in evoked amplitude when combined with VGlut overexpression. The conditions causing this phenotype included a double heterozygous loss-of-function condition for genes encoding the inositol trisphosphate receptor $\left(\mathrm{IP}_{3} \mathrm{R}-i t p r\right)$ and ryanodine receptor $(R y R) . \mathrm{IP}_{3} \mathrm{Rs}$ and RyRs gate calcium release from intracellular stores. Pharmacological agents targeting $I P{ }_{3} R$ and RyR recapitulated the genetic losses of these factors, as did lowering calcium levels from other sources. Our data are consistent with the idea that the homeostatic signaling process underlying PHD is especially sensitive to levels of calcium at the presynapse.

\section{Introduction}

Animal nervous systems use forms of homeostatic synaptic plasticity to maintain stable function. Over the last 20-25 years, studies from diverse systems have revealed a wealth of information about how forms of homeostatic synaptic plasticity are implemented (Davis, 2013; Davis and Müller, 2015; Delvendahl and Müller, 2019; Marder and Goaillard, 2006; Pozo and Goda, 2010; Turrigiano, 2008). In particular, the Drosophila melanogaster neuromuscular junction (NMJ) has uncovered many facets of homeostatic implementation on a molecular level (Frank, 2014a; Frank et al., 2020). Much of the NMJ homeostasis work in both Drosophila and vertebrates has focused on a form of homeostatic plasticity termed presynaptic homeostatic potentiation (PHP). With PHP, manipulations that impair postsynaptic muscle receptor function trigger an increase in presynaptic vesicle release (Cull-Candy et al., 1980; Davis et al., 1998; Frank et al., 2006; Petersen et al., 1997; Wang et al., 2016).

Homeostatic plasticity at the NMJ is a bi-directional process. First, PHP is reversible - when manipulations that impair muscle receptor function are removed, the presynaptic potentiation ceases (Wang et al., 2016; Yeates et al., 2017). Second, the Drosophila NMJ can depress quantal content in a homeostatic manner functionally opposite to PHP: Presynaptic homeostatic depression (PHD). Experimentally, one way to trigger PHD is to overexpress the Drosophila vesicular glutamate transporter gene, VGlut, in motor neurons. Overexpression of the glutamate transporter leads to an increase in the diameter of glutamatergic vesicles, an increase in quantal size across the entire distribution of spontaneous miniature events, and very large spontaneous quantal events (Daniels, 2004). To compensate for this, quantal content at the NMJ is lowered, resulting in normal evoked postsynaptic excitation (Daniels et al., 2004). 
Many genes have been shown to be necessary for PHP at the NMJ. But much less is known about PHD. PHP and PHD result in opposite changes in quantal content, and studies suggest divergent and separable mechanisms governing these forms of homeostatic plasticity. Some genes required for homeostatic potentiation are dispensable for homeostatic depression (Gaviño et al., 2015; Li et al., 2018; Marie et al., 2010). Moreover, unlike homeostatic potentiation, homeostatic depression does not appear to involve a change in the size of the readily releasable pool of synaptic vesicles (Li et al., 2018). Rather, homeostatic depression appears to involve a decrease in release probability (Gaviño et al., 2015). Finally, PHP at the NMJ appears to be a process that is dependent on the input (i.e. the type of synapse formed at the NMJ) (Newman et al., 2017) while PHD does not appear to be input specific (Li et al., 2018).

The degree of overlap between homeostatic depression and homeostatic potentiation is unknown. We designed a small-scale, directed screen to test for links between these two forms of homeostatic plasticity. For the screen, we targeted genes based on prior evidence that their impairment in the neuron caused a failure of the long-term maintenance of PHP. We examined loss-of-function conditions for these genes in a VGlut overexpression background for PHD. We did not find any cases of failed homeostatic depression - the conditions we examined showed decreases in quantal content in response to increased quantal size. However, we did find an interesting and unexpected evoked neurotransmission phenotype: a robust decrease in excitatory postsynaptic potential (EPSP) amplitude in a VGlut overexpression background. We observed this phenotype for a double heterozygous lossof-function condition for the Ryanodine and $\mathrm{IP}_{3}$ receptors. In our follow-up work, pharmacology phenocopied this genetic result, and our overall findings are consistent with the idea that the PHD system may show a heightened sensitivity to low calcium.

Our findings highlight a novel synaptic transmission phenotype. Prior characterizations of homeostatic depression do not report decreases in EPSP amplitude in VGlut overexpression relative to controls (Daniels et al., 2004; Gaviño et al., 2015; Li et al., 2018; Marie et al., 2010). Studies at the NMJ generally suggest models in which homeostatic compensation maintains evoked neurotransmission at the synapse approximately at control levels (Davis, 2013). Our results suggest that impairing store calcium channels may result in a cumulative defect in neurotransmission when there is a concurrent PHD challenge. We find this interesting, especially in light of the fact that these same store channels are required for the maintenance of PHP (James et al., 2019) and because other recent studies in other systems have implicated store calcium in presynaptic release mechanisms (e.g., (de Juan-Sanz et al., 2017)).

\section{Materials and Methods}

\section{Drosophila stocks and husbandry}

Fruit fly stocks were obtained from the Bloomington Drosophila Stock Center (BDSC, Bloomington, Indiana), Kyoto Stock Center (DGRC, Kyoto, Japan), Japan National Institute of Genetics (Mishima, Shizuoka, Japan), Vienna Drosophila Research Center (VDRC, Vienna, Austria), or from the labs that generated them. $w^{1118}$ was used as a wild-type (WT) control (Hazelrigg et al., 1984). RNAi lines and mutants used in the screen are reported in Supplemental Table S1.

Fruit flies were raised on cornmeal, molasses, and yeast medium (see BDSC website for standard recipe) in temperature-controlled conditions. Animals were reared at $25^{\circ} \mathrm{C}$ until they reached the wandering third instar larval stage, at which point they were selected for electrophysiological 
2006; Meyer and Aberle, 2006) to drive constitutive overexpression of VGlut. The full genotype of these animals is: $w$; VGlut, OK371-Gal4/CyO-GFP. Virgins of these flies were crossed to RNAi lines or mutants to test for changes to homeostatic depression. $w$; OK371-Gal4/+ was used as a genetic control for baseline electrophysiology.

\section{Electrophysiology and analysis}

Larvae were dissected in a modified HL3 saline comprised of: $\mathrm{NaCl}(70 \mathrm{mM}), \mathrm{KCl}(5 \mathrm{mM}), \mathrm{MgCl}_{2}$ $(10 \mathrm{mM}), \mathrm{NaHCO}_{3}(10 \mathrm{mM})$, sucrose $(115 \mathrm{mM}=3.9 \%)$, trehalose $(4.2 \mathrm{mM}=0.16 \%)$, HEPES $(5.0$ $\mathrm{mM}=0.12 \%)$, and $\mathrm{CaCl}_{2}(0.5 \mathrm{mM}$, except as noted $)$.

For pharmacology, Dantrolene (R\&D Systems) and Xestospongin C (Abcam) were used. Dantrolene was mixed into saline to a final concentration of $25 \mu \mathrm{M}$. Larvae were cut open on the dorsal side and allowed to incubate in the Dantrolene saline for 5 minutes. The rest of the dissection and recording was completed in Dantrolene saline. Xestospongin $\mathrm{C}$ was applied in a similar manner, with the animals allowed to incubate in $20 \mu \mathrm{M}$ Xestospongin $\mathrm{C}$ saline for 5 minutes before they were recorded, also in saline containing Xestospongin $\mathrm{C}$.

Electrophysiological data were collected using an Axopatch 200B amplifier (Molecular Devices, Sunnyvale, CA) in bridge mode, digitized using a Digidata 1440A data acquisition system (Molecular Devices), and recorded with pCLAMP 10 acquisition software (Molecular Devices). A Master-8 pulse stimulator (A.M.P. Instruments, Jerusalem, Israel) and an ISO-Flex isolation unit (A.M.P. Instruments) were utilized to deliver $1 \mathrm{~ms}$ suprathreshold stimuli to the appropriate segmental nerve. The average spontaneous miniature excitatory postsynaptic potential (mEPSP) amplitude per NMJ was quantified by hand, approximately 100 individual spontaneous release events per NMJ (MiniAnalysis, Synaptosoft, Fort Lee, NJ). Measurements from all NMJs of a given condition were then averaged. For evoked neurotransmission, 30 excitatory postsynaptic potentials (EPSPs) were averaged to find a value for each NMJ. These were then averaged to calculate a value for each condition. Quantal content (QC) was calculated by the ratio of average EPSP and average mEPSP amplitudes for each individual NMJ. An average quantal content was then calculated for each condition. EPSP variability was assessed by measuring each of the 30 traces individually and calculating a standard deviation and range for that NMJ. Range was defined as the maximum EPSP value minus the minimum EPSP value.

\section{Immunostaining}

An immunostaining experiment is detailed for Figure 4. Procedures match those previously published (Brusich et al., 2015; Brusich et al., 2018; James et al., 2019; Spring et al., 2016; Yeates et al., 2017). Briefly, third instar larvae were filleted and fixed for 5 minutes with Bouin's fixative (Ricca Chemical, Arlington, TX). After washes, fixed fillets were incubated in primary antibodies overnight at $4^{\circ} \mathrm{C}$, mouse anti-Brp (nc82, 1:250, University of Iowa Developmental Studies Hybridoma Bank) (Wagh et al., 2006) and rabbit anti-Dlg (1:5,000) (Budnik et al., 1996). After washes, fillets were incubated in fluorophore-conjugated secondary antibodies overnight at $4^{\circ} \mathrm{C}$ (Jackson ImmunoResearch Labs, West Grove, PA), goat anti-mouse-488 (DyLight, 1:1000) and goat anti-rabbit-549 (DyLight, 1:2000). After washes, fillets were mounted and Dlg boutons were counted blinded by hand and double checked for Brp signal in apposition.

\section{Statistical Analyses}

Statistical analyses were conducted using GraphPad Prism Software. Statistical significance was assessed either by Student's T-Test when one experimental data set was being directly compared to a 
control data set, or one-way ANOVA with Tukey's post-hoc test when multiple data sets were being compared. Specific $p$ value ranges are noted in the Figure legends and shown in graphs as follows: ${ }^{*} p$ $<0.05, * * p<0.01$, and $* * * p<0.001$ ( $*$ and \# are used in Figures if there are additional comparisons highlighted). For some comparisons that are close to $p<0.05$ statistical significance but do not achieve it $(0.05<p<0.1)$, specific values are reported on the graph itself. Calcium cooperativity data were analyzed using a non-linear fit regression analysis on GraphPad Prism.

\section{$1473 \quad$ Results}

\section{A recombinant line to analyze presynaptic homeostatic depression (PHD)}

149 Using previously published reagents, we generated a fly stock with constitutive VGlut transgene overexpression. Such a stock could be used as a tool for a single-cross genetic screen. To generate the stock, we recombined the OK371-Gal4 motor neuron driver (Mahr and Aberle, 2006; Meyer and Aberle, 2006) with a UAS-VGlut transgene (Daniels et al., 2004). We placed these two genetic elements in cis on Drosophila melanogaster Chromosome II. OK371-Gal4 is an enhancer trap line for the VGlut promoter itself. This ensured that GAL4-driven $U A S$-VGlut overexpression would happen in desired tissues, Drosophila motor neurons.

We tested if the recombinant line constitutively overexpressing $U A S$-VGlut could express PHD at the NMJ. We crossed the recombinant stock to our wild-type stock ( $w^{1118}$, herein: WT) (Cross result, herein: "VGlut, OK371/+"). By NMJ electrophysiology, we recorded from WT control, OK371/+ control, and w; VGlut, OK371/+. As expected, VGlut, OK371/+ NMJs showed an increase in spontaneous miniature excitatory postsynaptic potential (mEPSP) amplitude compared to controls (Fig. 1A-C; data also in Supplementary Table S1). Compared to WT control NMJs, there was no significant difference in evoked postsynaptic amplitudes for VGlut, OK371/+ NMJs (Fig. 1D; $p=0.82$, one-way ANOVA). This was because of an accurate homeostatic decrease in quantal content (QC) (Fig 1E) hence, successful PHD. This result matched prior studies that had used WT as a control and a trans OK371/UAS-VGlut combination to induce PHD (Daniels et al., 2004; Gaviño et al., 2015; Li et al., 2018).

167 Even though PHD was successful relative to WT for our test cross, we noted a small, but statistically 168 significant, baseline increase in the EPSP amplitude of OK371/+ NMJs. This increase in $O K 371 /+$ EPSP level was present compared either to WT control or to VGlut, OK371/+ (Fig. 1D). One possibility is that the $O K 371 /+$ genetic background has slightly elevated release, and the combined addition of $U A S$-VGlut reveals a slight depression in evoked amplitude. Noting this potentially important difference in our driver control, we continued using the $O K 371 /+$ heterozygous condition as a genetic background control. OK371/+ is a closer genetic control for PHD analysis than WT.

\section{A genetic screen identifies an interaction between calcium stores and a PHD-inducing challenge}

We used our recombinant line to conduct a genetic screen for conditions that affect presynaptic homeostatic depression (PHD). We crossed this stock to screen stocks: 1) either to drive UAS-RNAi transgenes to knock down genes; 2) to drive other chosen $U A S$ transgenes; or 3) to combine with $\backslash$ heterozygous loss-of-function mutant lines (Materials and Methods, Fig. 2A). For the screen, we targeted a subset of genes previously identified as in the neuron for homeostatic potentiation, or closely related genes. We tested 43 genotypes (sometimes multiple conditions for a single gene), including our homeostatic depression condition, VGlut, OK371/+ (Fig. 2B, C). 
The aggregate results of the screen are reported here (Fig. 2B, C; raw data in Supplementary Table S1). We recorded from 42 experimental heterozygous mutant/+ or $>U A S$-RNAi or -transgene/+ conditions, in the VGlut, OK371/+ genetic background. Of those 42, 12 achieved EPSPs that were numerically larger than VGlut, $O K 371 /+$, and 22 achieved QCs that were numerically larger than $V G l u t, O K 371 /+$ (Fig. 2B, C). Increased evoked potentials could signify failed PHP - however, none of these cases represented statistically significant increases compared to VGlut, OK371/+. None were so much bigger that they were good candidates for "failed PHD." Indeed, all of the candidates had average EPSP and QC levels below OK371/+ NMJ baseline recordings (Compare Figs 1D, E and Fig. 2B, C).

We noted a phenotype distinct from what we were initially seeking: two crosses yielded larvae with striking decreases in NMJ EPSP amplitudes, more than two standard deviations below the average EPSPs from the baseline VGlut, OK371/+ data set (Fig. 2B). One case was knockdown of the Survival motor neuron (Smn) gene with the UAS-Smn[RNAi] $]^{J F 02057}$ line in the VGlut, OK371/+ background. This was intriguing because Drosophila Smn is homologous to human SMN. Defects in SMN cause Spinal Muscular Atrophy (Lefebvre et al., 1995). Drosophila Smn has been characterized as a potential model for Spinal Muscular Atrophy (Raimer et al., 2020; Sen et al., 2011; Spring et al., 2019). Smn has also previously been implicated in PHP (Sen et al., 2011). However, the result for UAS$S m n[R N A i]^{J F 02057}$ was not replicated by other Smn knockdown or loss-of-function mutant test crosses (Fig. 2B, C). We did not follow up on Smn for this study.

A second case with a striking decrease in EPSP amplitude in the screen was a double heterozygous genetic condition in genes encoding the Drosophila Ryanodine receptor $(R y R)$ and inositol 1,4,5trisphosphate $\left(\mathrm{IP}_{3)}\right.$ receptor (itpr): VGlut, $O K 371 / R y R^{E 4340 K}$; itpr ${ }^{90 B} /+$ (Fig. 2B, C). Ryanodine receptors (RyRs) and $\mathrm{IP}_{3}$ receptors $\left(\mathrm{IP}_{3} \mathrm{Rs}\right)$ are localized to the endoplasmic reticulum. They mediate release of calcium from intracellular stores (Berridge, 1984, 1987, 1998; Simkus and Stricker, 2002). The $R y R^{E 4340 K}$ mutation is a single amino acid substitution (glutamic acid to lysine) (Dockendorff et al., 2000), and the itpr ${ }^{90 B}$ mutation is a null mutant generated by imprecise excision of a transposon (Venkatesh and Hasan, 1997). We previously defined roles for RyR, $\mathrm{IP}_{3} \mathrm{R}, \mathrm{IP}_{3}$ signaling and upstream components in maintaining presynaptic homeostatic potentiation (PHP) (Brusich et al., 2015; James et al., 2019).

In parallel, we screened single mutant manipulations for both genes. Neither the $R y R^{E 4340 K /+}$ heterozygous condition, nor the $i t^{90 B} /+$ heterozygous condition - nor any single heterozygous or RNAi knockdown condition for either gene - yielded as significantly depressed EPSPs in response to PHD challenge (Fig. 2B, C). Therefore, the screen result with the double heterozygote could be due to a genetic interaction, or it could be due to other factors in the genetic background. This preliminary finding required further characterization.

We tested if the electrophysiological phenotype could be due to a baseline neurotransmission defect when both genes are heterozygous. By electrophysiology, we compared NMJs from $O K 371 / R y R^{E 4340 K \text {; }}$ $i t p r^{90 B} /+$ larvae as a baseline double heterozygous condition vs. NMJs from $V G l u t, O K 371 / R y R^{E 4340 K}$; $i t p r^{90 B} /+$ larvae (Fig. 3A-D). Just like WT, the baseline double heterozygous condition did have a slight decrease in EPSP amplitude compared to OK371/+ driver control (Fig. 3A). This indicated a small, but discernible defect in neurotransmission in animals where the IP ${ }_{3}$ Rs and RyRs are both impaired. The double heterozygous condition with concurrent VGlut gene overexpression showed a further decrease in transmission - compared to its own genetic control, it had increased quantal size (Fig. 3B), but significantly decreased evoked amplitude (Fig. 3C) because of a large decrease in quantal content (Fig. 3D). Finally, the quantal content for VGlut, OK371/Ry $R^{E 4340 K}$; itpr $r^{90 B} /+$ NMJs was numerically smaller than for VGlut, OK371/+ NMJs (Fig. 3D), but this latter numerical difference was not statistically significant ( $p=0.07$, one-way ANOVA). 
We noted that the EPSP amplitude in individual VGlut, OK371/RyR $R^{E 4340 K} ; i t p r^{90 B} /+$ NMJ recordings varied markedly from stimulus to stimulus. High variability could indicate unstable neuronal excitability or release. To check if evoked release events were indeed more variable, we completed additional analyses. First, we extracted the amplitude of each individual EPSP event at every NMJ recorded. From these data, we calculated the EPSP standard deviation (S.D.) and coefficient of variation (C.V.) per individual NMJ. We also calculated a range for each NMJ by subtracting the maximum EPSP of the thirty from the minimum. We averaged these S.D., C.V., and range measures for each genotype, considering all of the individual EPSP recordings. For all of these EPSP parameters, w; VGlut, $O K 371 / R y R^{E 4340}$; itpr $r^{90 B} /+$ animals showed statistically significant higher variability compared to controls (Fig. 3E). By contrast, double heterozygous baseline $O K 371 / R y R^{E 4340 K}$; itpr $r^{90 B} /+$ NMJs did not differ significantly from $w$; OK371/+ driver control NMJs $(p>0.85$ for each measure, Kruskal-Wallis ANOVA), suggesting that the variability stems from VGlut overexpression in the mutant background (Fig. 3E). w; VGlut, OK371/+ NMJs showed numerically higher variability than $w$; OK371/+, but this was not statistically significant (Fig. 3E, $p>0.25$ for each measure, KruskalWallis ANOVA).

Finally, we conducted immunostaining to check if any of these electrophysiological defects might correspond with defects in synaptic growth. We assessed growth by co-staining with antibodies against the postsynaptic PSD-95 homolog, DLG (Budnik et al., 1996) and the presynaptic active zone protein, BRP (Wagh et al., 2006). We counted boutons encased by anti-DLG signal and checked that these boutons were apposed by anti-BRP signal. By this analysis, we saw no significant changes in NMJ growth: neither the PHD challenge; nor the double heterozygous loss of the $R y R /+$ and itpr/+ genes; nor combining those manipulations together yielded significant numerical differences in bouton count ( $p>0.90$ for all comparisons, one-way ANOVA; Fig 4A, B). One caveat to these results is that we only examined these NMJs at the level of bouton count, not at the level of the abundance of specific active zone markers (as in (Böhme et al., 2019; Goel et al., 2019; Gratz et al., 2019)).

\section{Pharmacology targeting Ryanodine and $I P_{3}$ receptors recapitulates loss-of-function genetics}

We tested if the electrophysiological phenotypes we observed could be recapitulated by combining genetics and pharmacology. We started with the drug Dantrolene. Dantrolene is a RyR antagonist (Vazquez-Martinez et al., 2003; Zhao et al., 2001). In prior work at the Drosophila NMJ, we found that application of Dantrolene can abrogate the long-term maintenance of PHP (James et al., 2019). We used a sensitized $O K 371 /+$; itpr ${ }^{90 B} /+$ genetic background. With this background, we could pharmacologically impair RyRs while also genetically impairing $\mathrm{IP}_{3} \mathrm{Rs}$. We applied $25 \mu \mathrm{M}$ of Dantrolene to: 1) $O K 371 /+$ NMJs; 2) VGlut, OK371/+ NMJs; 3) $O K 371 /+$; $i t p r r^{90 B /+}$ NMJs; and 4) VGlut, $O K 371 /+$; itpr ${ }^{90 B} /+$ NMJs. We also collected a set of data for genetically identical conditions without drug treatment (Fig. 5A).

In the absence of drug treatment, PHD proceeded normally (Fig. 5A). We noted that the untreated OK371/+; itpr $90 B /+$ heterozygous condition had a slightly diminished evoked amplitude compared to OK371/+ (Fig. 5A, middle). Therefore, the $i t p r^{90 B} /+$ condition could be contributing some neurotransmission loss on its own. But in this case, the addition of VGlut transgenic expression to this heterozygous background did not further decrease evoked neurotransmission (Fig. 5A, middle), indicating normal PHP.

With $25 \mu \mathrm{M}$ Dantrolene treatment, we noted that the mEPSP amplitudes were generally smaller than without treatment (compare Figs. 5A, B). Nevertheless, in the VGlut-overexpressing backgrounds, mEPSPs were still elevated compared to their respective controls (Fig. 5B). This indicated that in the 
Dantrolene, EPSP amplitudes in VGlut-overexpressing lines were significantly decreased compared to their respective genetic controls (Figs. 5B, C). This was because of a marked decrease in QC (Fig. 5B). In particular, the VGlut, $O K 371 /+$; tppr $^{90 B} /+$ condition (+ Dantrolene) had depressed evoked amplitudes compared either to the VGlut, OK371/+ (+ Dantrolene) condition or to the OK371/+; itpr $r^{90 B} /+(+$ Dantrolene) condition (Figs. 5B, C). Collectively, these data could indicate a cumulative neurotransmission defect when impairing both the $\mathrm{IP}_{3}$ Rs and RyRs in a PHD-challenged background.

It is possible that strong impairment of RyRs could be sufficient to cause synthetic phenotypes in conjunction with the PHD regulation system. We ran additional pharmaco-genetic tests using a second sensitized genetic background, $O K 371 / R y R^{E 4340 K}$ - both with and without drugs and with and without $U A S$-VGlut overexpression. Again, in the absence of pharmacological treatment, PHD proceeded normally in the heterozygous $O K 371 / R y R^{E 4340 K}$ genetic background (Fig. 6A). With Dantrolene, mEPSPs became significantly larger when VGlut was expressed (Fig. 6B, left), but EPSPs were significantly reduced (Figs. 6B, middle, 6D) because of a decrease in quantal content (Fig. 6B, right).

Finally, we attempted the inverse pharmaco-genetic experiment from that in Figure 5. This time we used the $\mathrm{IP}_{3} \mathrm{R}$ inhibitor, Xestospongin C (Gafni et al., 1997; Wilcox et al., 1998) and the sensitized $O K 371 / R y R^{E 4340 K}$ genetic background. We applied $20 \mu \mathrm{M}$ Xestospongin $\mathrm{C}$, both to $O K 371 / R y R^{E 4340 K}$ NMJs and to VGlut, OK371/RyR $R^{E 4340 K}$ NMJs. mEPSPs were numerically larger when VGlut was overexpressed (Fig. 6C, left) - though interestingly, for the Xestospongin $\mathrm{C}$ dataset, the data did not achieve statistical significance for mEPSP size ( $p=0.10$, one-way ANOVA). This could indicate only weak to no homeostatic pressure in the presence of Xestospongin C. Nevertheless, EPSPs were significantly reduced (Figs. 6C, middle, 6D) because of a marked decrease in quantal content (Fig. 6C, right).

Taking all of these data together, for each case where we examined a dual impairment of RyR and IP $\mathrm{IP}_{3}$ the EPSP amplitudes were all quite low with concomitant VGlut overexpression (Figs. 3, 5, 6).

\section{PHD in very low extracellular calcium}

300 We wondered how impairment of channels that mediate release of calcium from intracellular stores might cause the electrophysiological phenotypes that we observed. It could be the case that they are part of the PHD system. Or it could be the case that impairing these channels does not impinge upon PHD signaling itself - but their loss may sensitize the synapse to additional challenges, such as those brought on by PHD.

305 Our prior work suggested that these ER calcium store channels and the signaling systems that control them are required to maintain homeostatic potentiation throughout life (Brusich et al., 2015; James et al., 2019). We also found a related result: impairing $\mathrm{Ca}^{2+}$ store release mollified hyperexcitability phenotypes caused by gain-of-function Cav2 amino-acid substitutions in the alpha1 subunit Cacophony. Cav2 channels mediate synaptic calcium influx at the NMJ (Brusich et al., 2018). In light of these prior data, we considered two possibilities for PHD. One model is that the $\mathrm{IP}_{3} \mathrm{R}$ and RyR channels play a role in ensuring proper level of neurotransmission coincident with PHD. A different model is that calcium itself plays the important role. If this latter idea were true, it might be the case that lowering calcium influx into the presynaptic terminal would also be sufficient to interact with the PHD signaling process, ultimately lowering evoked transmission.

As a test, we measured release over a range of low extracellular calcium concentrations $(0.2-0.5 \mathrm{mM})$. We examined six genotypes: 1) WT; 2) $w$; OK371/+ ; 3) $w$; VGlut, OK371/+; 4) $w$; $R y R^{E 4340 K /+ \text {; }}$ itpr $r^{90 B} /+$; 5) w; OK371/RyR $R^{E 4340 K}$; itpr $r^{90 B /+}$; and 6) w; VGlut, OK371/RyR $R^{E 4340 K}$; itpr $r^{90 B /+}$. To organize data and to calculate calcium cooperativity, we plotted quantal content as a function of 
calcium concentration, with the $\mathrm{x}-\mathrm{y}$ axes on a $\log -\log$ scale (Fig. 7A, B). To account for different $\mathrm{Ca}^{2+}$ driving forces in the different concentrations, we corrected QC for nonlinear summation in our plots and in our subsequent analyses (NLS Corrected QC) (Martin, 1955).

Non-linear regression analyses revealed that there was no significant difference in calcium cooperativity between any of these genotypes over the range of extracellular $\left[\mathrm{Ca}^{2+}\right]$ we tested (Fig. 7A, B). The calculated log-log slope values of the control PHD genotypes were: WT $(\log -\log$ slope $=$ $1.810), w ; O K 371 /+(\log -\log$ slope $=1.884)$, and $w ; V G l u t, O K 371 /+(\log -\log$ slope $=2.117)$. Comparing those three slopes with one another by nonlinear regression yielded no significant difference in slope $(p=0.91)$. The log-log slope values of the double heterozygous conditions were: $w ; R y R^{E 4340 K} /+;$ itpr $^{90 B} /+(\log -\log$ slope $=1.737), w ; O K 371 / R y R^{E 4340 K} ;$ itpr $^{90 B} /+(\log -\log$ slope $=$ 2.102), and $w$; VGlut, $O K 371 / R y R^{E 4340 K} ; i t p r^{90 B} /+(\log -\log$ slope $=1.601)$. Comparing those slopes with one another also yielded no significant difference $(p=0.77)$.

Even though there was no significant difference in calcium cooperativity of release over the range of low $\left[\mathrm{Ca}^{2+}\right]$ conditions examined, our data did show a very large drop in release between 0.3 and 0.2 $\mathrm{mM}\left[\mathrm{Ca}^{2+}\right]$ - specifically for the genotypes where PHD was induced by $U A S$-VGlut overexpression, or for the genotypes with a double heterozygous impairment of $R y R$ and itpr. Examining the raw data at $0.2 \mathrm{mM}\left[\mathrm{Ca}^{2+}\right]$, we observed that there was significant homeostatic pressure for PHD signified by mEPSP amplitude increases in the VGlut-overexpression background (Fig. 7C, left). Yet except for the control NMJs, EPSP amplitudes were very much diminished (Fig. 7C, middle) because of stark drops in QC (Fig. 7C, right).

Together, the data point to two conclusions. First, low extracellular calcium on its own appears to be a case where the synapse experiences a synergistic interaction with PHD challenge (Fig. 7C, VGlut, $O K 371 /+$ data). Second, double heterozygous impairment of $R y R$ and itpr appears to cause very low levels of baseline release in low calcium, irrespective of PHD challenge (Fig. 7C, middle; compare with Fig. 3C). Taken together, these data suggest that lowering presynaptic calcium by any means (impairing store release and/or impairing influx) is sufficient to impair evoked levels of excitation, in conjunction with a PHD challenge.

\section{PHD challenge interacts with impaired $\mathrm{Ca}_{V} 2$ function}

As a final test, we turned back to genetics. Drosophila $\mathrm{Ca}_{\mathrm{v}} 2$ channels mediate synaptic calcium influx at the NMJ. We used a hypomorphic mutant in the Cav2 alpha1 subunit-encoding cacophony gene, $c a c^{S}$, to limit calcium influx. Cav2 is essential for viability, but $c a c^{S}$ hypomorphs are viable and fertile (Kawasaki et al., 2000; Smith et al., 1998). Prior work showed that the $c a c^{S}$ homozygous condition dampens NMJ EPSP amplitude by about 70-80\% (Frank et al., 2006); calcium imaging data suggest this is due to a $\sim 50 \%$ decrease in $\mathrm{Ca}^{2+}$ influx during evoked stimulation (Müller and Davis, 2012). Beyond this phenotype in baseline neurotransmission, $\mathrm{cac}^{S}$ hypomorphs also block PHP expression and PHP-associated increases in presynaptic calcium influx (Frank et al., 2006; Müller and Davis, 355 2012).

With a single cross, we generated hemizygous $c a c^{S} / Y$; VGlut, OK371/+ male larvae (Fig. 8A). Compared to $c a c^{S} / Y$ as a baseline mutant control, $c a c^{S} / Y$; VGlut, OK371/+ NMJs have a marked increase in mEPSP size (Fig. 8B), indicating homeostatic pressure to induce PHD (Fig. 7B). However, comparing evoked potentials of those two conditions shows that $c a c^{S} / Y$; VGlut, OK371/+ NMJs have much smaller EPSPs (Fig. 8C) and a very large decrease in QC (Fig. 8D). 
We began this study in search of genetic conditions that affect PHD (Fig. 2). While we did not find any conditions that result in a block of PHD, we did find conditions that provide insight into how calcium regulation may interact with this form of homeostatic plasticity to affect synapse function. When $\mathrm{IP}_{3} \mathrm{R}$ and RyR functions are partially impaired - either by genetics or by pharmacology - the NMJ still executes a PHD-like process. But that process goes beyond what is appropriate for the homeostatic pressure that is applied to the system. As a result, evoked potentials at the NMJ are much smaller than baseline (Figs. 3, 5, 6). A similar phenotype is observed when extracellular $\left[\mathrm{Ca}^{2+}\right]$ is lowered to 0.2 $\mathrm{mM}$ (Fig. 7) and when the Cav2 alpha1 subunit gene cacophony harbors a hypomorphic mutation, $\mathrm{cac}^{S}$ (Fig. 8).

This phenotype has important implications for proper control of synapse function. Taking our data together, we propose that perturbations that dampen calcium efflux from stores or perturbations that dampen calcium influx from the extracellular environment can both synergistically interact with a PHD challenge to control levels of evoked neurotransmission (Fig. 9).

\section{Screen limitations}

We did not identify conditions that blocked PHD, and here we discuss potential limitations of the screen. First, our primary assay was electrophysiology, and we employed a candidate-based method, similar to what has previously been documented in the field for PHP (Frank et al., 2020). By definition, candidate-based screens are limited in scope. Second, we focused on factors previously implicated in the maintenance of PHP function (or closely related signaling factors). The idea was that some factors needed to maintain synaptic homeostasis may be needed to orient the NMJ toward a proper, physiological level of function, regardless of the nature of the homeostatic challenge. This idea could have valence, but it was not guaranteed to produce mutant conditions with greater than normal evoked amplitudes in our screen.

Regarding the electrophysiological data, we did find instances in which the screened EPSP was numerically larger than the baseline for VGlut, OK371/+, but no instances identified as "PHDblocking" (Fig. 2). The VGlut, OK371/+ baseline evoked potential was high $(\sim 35 \mathrm{mV})$, so it is possible that potential positives at a higher potential could be obscured by the limits of non-linear summation. There were also variations from line to line in resting membrane potential, input resistance, and the degree of mEPSP increase indicative of PHD challenge (Supplementary Table, S1). All of these parameters could contribute to false negatives for the screen. Unless a screen is done to saturation, there will be false negatives. It is important to interpret those parsimoniously. For our screen, we believe the way to interpret a negative is not to state that the screen definitively ruled out a factorrather, the screen failed to rule in that factor for follow-up study.

\section{Similarities and differences with prior PHD studies at the NMJ}

We were able to conduct a PHD screen using our recombinant stock with the UAS-VGlut and OK371Gal4 elements on the same chromosome. In principle, such a stock can pick up modifier mutations. The trade-off was a simplified, single-generation crossing scheme for genetic screens. Our recombinant stock with the driver and $U A S$ elements in cis maintains consistent PHD challenge from generation to generation, and it behaves similarly electrophysiologically to trans OK371/VGlut combinations used in other studies (Daniels et al., 2004; Gaviño et al., 2015; Li et al., 2018).

There are differences between our study and the findings of other published work. Prior studies have used WT (or $w^{1118}$ ) as a control background when compared to VGlut overexpression (Daniels et al., 2004; Gaviño et al., 2015; Li et al., 2018). This is a standard practice. Those studies reported precise 
PHD when comparing WT vs. OK371/VGlut third instar larvae - decreased QC at OK371/VGlut NMJs resulting in unchanged evoked transmission. We replicated this finding (Fig. 1). However, we also used our Gal4 driver stock background $O K 371 /+$ as an additional control. For that comparison, we saw a slight depression in the evoked amplitude of OK371, VGlut/+ NMJs (Fig. 1). One possibility is that our recombinant stock was acting as a sensitized background.

A second difference comes from the low extracellular calcium test. A low extracellular calcium experiment was previously done when VGlut overexpression was first characterized (Daniels et al., 2004). For that study, the authors showed that QC was significantly diminished compared to wild-type NMJs by the method of failure analysis. Taking the data of that study in aggregate, the authors concluded that PHD was intact in a variety of conditions, including saline with very low extracellular $\left[\mathrm{Ca}^{2+}\right]\left(0.23 \mathrm{mM} \mathrm{Ca}^{2+}, 20 \mathrm{mM} \mathrm{Mg}{ }^{2+}\right)$. Our study may appear to conflict with that study because we found that saline with very low $\left[\mathrm{Ca}^{2+}\right]\left(0.2 \mathrm{mM} \mathrm{Ca}^{2+}, 10 \mathrm{mM} \mathrm{Mg}^{2+}\right)$ is conducive an interaction with PHD, resulting in low evoked release. One possibility is that since the original study was examining failure percentage vs. WT - and not the absolute value of mEPSPs or EPSPs in low calcium, this might not be as easily observed. Other differences might be attributed genetic background or other differences in recording saline, like magnesium concentration.

Finally, one other study previously examined the effects of a $c a c^{S}$ mutation with concomitant VGlut overexpression (Gaviño et al., 2015). The authors did not find the low evoked potentials that we report. The major difference between that experiment and ours is that the prior work examined the $\mathrm{cac}^{S}$ mutation in an extracellular $\left[\mathrm{Ca}^{2+}\right](1.0 \mathrm{mM})$ that was double that of our study. The result was a $\mathrm{Ca}^{2+}$ driving force that yielded robust baseline EPSPs, even in the $c a c^{S}$ mutant background (Gaviño et al., 2015). Given our results with low calcium concentration (Fig. 7), a similar effect may be at work here.

\section{Known roles for calcium in controlling homeostatic plasticity}

432 The notion that calcium contributes to successful homeostatic signaling is not new. Many roles for voltage-gated calcium channels in synaptic homeostasis are well-documented (Frank, 2014a, b). Prior to our study, there was evidence for voltage-gated calcium channel regulation for both NMJ PHP and PHD. For PHP, loss-of-function conditions in Cav2/cacophony can impair or block this form of homeostatic regulation (Frank et al., 2006; Frank et al., 2009; Müller and Davis, 2012; Spring et al., 2016). Calcium imaging experiments suggest that the reason is because an increase in calcium influx through Cav2 is required for the upregulation of quantal content during PHP, and mutant conditions like $c a c^{S}$ block this increase (Müller and Davis, 2012). Recent studies report that Cacophony and other active zone protein levels increase at the NMJ active zone in response to PHP homeostatic challenges (Böhme et al., 2019; Goel et al., 2019; Gratz et al., 2019). And work from mammalian systems mirrors these findings. For example, with mouse hippocampal cultures, TTX exposure induces a homeostatic decrease in presynaptic calcium influx (Zhao et al., 2011).

The converse appears true for PHD. Calcium imaging data from two different studies has shown a decrease in the size of calcium transients at the NMJ in response to presynaptic nerve firing in VGlutoverexpressing animals (Gaviño et al., 2015; Li et al., 2018). The data are mixed on how these decreased transients might come about during PHD. Using a tagged UAS-cacophony cDNA transgene, two studies verified that there was a reduction in the amount of GFP-tagged Cacophony alpha1 subunits in Cav2 in a VGlut-overexpressing background (Gaviño et al., 2015; Gratz et al., 2019). However, one of these same studies demonstrated that if a tagged genomic construct is used instead, that same Cav2 reduction is not observed (Gratz et al., 2019). Since the transgenic tagged Cacophony-GFP is the 
case that some isoforms are more dynamically trafficked at the synapse. Another possibility is that existing active zone components are somehow modulated during PHD. Regardless of the actual mechanism, the phenomenon appears conserved: again, with rodent hippocampal preparations, increased neuronal activity through gabazine exposure induces a PHD-like phenomenon ultimately resulting in decreases in calcium influx and release (Jeans et al., 2017; Zhao et al., 2011).

\section{How do calcium stores interact with PHD?}

460 Calcium stores have been studied in the context of neurotransmission and plasticity. We know that endoplasmic reticulum (ER) can be visualized at Drosophila NMJ terminals (Summerville et al., 2016), and recently developed imaging tools employed in multiple systems (including at the Drosophila NMJ) show how nerve stimulation results in dynamic changes to ER lumenal calcium, (de Juan-Sanz et al., 2017; Handler et al., 2019; Oliva et al., 2020). In parallel, other groups working at the Drosophila NMJ have demonstrated important roles in baseline neurotransmission and in PHP for ER resident proteins (Genç et al., 2017; Kikuma et al., 2017). And from our prior work, we know that store calcium channels and upstream signaling components are important for maintaining the NMJ's capacity for PHP throughout life (Brusich et al., 2015; James et al., 2019). We also know that disrupting these same factors can ameliorate hyperexcitability associated with gains of Cav2 function (Brusich et al., 2018). Finally, from mammalian work it is clear that $\mathrm{IP}_{3} \mathrm{Rs}$, RyRs, and intracellular calcium govern a variety of forms of neuroplasticity (Berridge, 2016), including paired pulse facilitation (Emptage et al., 2001), and modulation of voltage-gated calcium channel activity (Catterall, 2011; Lee et al., 2000).

If PHD were simply a matter of properly functioning neurotransmission machinery, then it is not entirely obvious why PHD would be so sensitive to the amount of calcium available such that evoked release would be impaired greatly either when store-operated channels were impaired or when the amount of influx was lowered. In our study, neurotransmission has not been lowered beyond a point of synapse failure. This means that there is still functional machinery. And PHD, per se, is not disrupted - indeed, there is still depression.

With any type of homeostatic system, there not only needs to be error detection (large quantal size) and correction (decreased quantal content), but there also need to be brakes applied to the system to prevent some kind of overcorrection. At first glance, our data could suggest some manner of PHD "overcorrection." In our view, this is an interesting and understudied type of phenomenon that could be examined in many homeostatic systems. But it is also true that the nature of the PHD challenge could simply represent a genetic background that renders the synapse sensitive to any additional insults.

So how exactly do levels of calcium (or the function of distinct types of calcium channels found at the synapse) ultimately affect excitation levels? This is a difficult problem. The first step might be to narrow the relevant tissue type(s) involved in PHD signaling. ER and store-operated channels are relevant to the functions of many tissues. In principle, our genetic loss-of-function manipulations to itpr and $R y R$ could affect store-operated channels either in the neuron or in the muscle or in surrounding tissues like glia. Our pharmacological manipulations using Dantrolene and Xestospongin $\mathrm{C}$ could also affect multiple tissue types. Therefore, in principle, changing the levels of cytosolic calcium could either affect local signaling in the neuron, or it could result in aberrant signaling back to the presynaptic neuron, disorienting the homeostat.

We favor the idea that the relevant calcium signal is local in the motor neuron for two reasons. First, from our own data, we were able to observe the small evoked neurotransmission phenotype either with manipulations to store calcium or with manipulations that affect presynaptic calcium influx, including partial loss-of-function of neuronal cacophony. Second, a recent study puts forth data suggesting that when VGlut overexpression induces PHD, this happens exclusively because of excess presynaptic 
glutamate release, and presynaptic depression is initiated independent of any sort of postsynaptic response (Li et al., 2018). Such an autocrine signaling mechanism could very well reveal a role for intracellular calcium signaling in the presynapse.

\section{$5 \quad$ References}

503 Berridge, M.J. (1984). Inositol trisphosphate and diacylglycerol as second messengers. Biochem J 220, 345-360.

505 Berridge, M.J. (1987). Inositol trisphosphate and diacylglycerol: two interacting second messengers. 506 Annu Rev Biochem 56, 159-193.

507 Berridge, M.J. (1998). Neuronal calcium signaling. Neuron 21, 13-26.

Berridge, M.J. (2016). The Inositol Trisphosphate/Calcium Signaling Pathway in Health and Disease. Physiol Rev 96, 1261-1296.

Böhme, M.A., McCarthy, A.W., Grasskamp, A.T., Beuschel, C.B., Goel, P., Jusyte, M., Laber, D.,

511 Huang, S., Rey, U., Petzoldt, A.G., et al. (2019). Rapid active zone remodeling consolidates

512 presynaptic potentiation. Nat Commun 10, 1085.

513 Brusich, D.J., Spring, A.M., and Frank, C.A. (2015). A single-cross, RNA interference-based genetic tool for examining the long-term maintenance of homeostatic plasticity. Frontiers in cellular neuroscience 9, 107.

517 Drosophila CaV2 channels harboring human migraine mutations cause synapse hyperexcitability that

518 can be suppressed by inhibition of a Ca2+ store release pathway. PLoS genetics 14, e1007577.

519 Budnik, V., Koh, Y.H., Guan, B., Hartmann, B., Hough, C., Woods, D., and Gorczyca, M. (1996).

520 Regulation of synapse structure and function by the Drosophila tumor suppressor gene dlg. Neuron

$521 \quad 17,627-640$.

522 Catterall, W.A. (2011). Voltage-gated calcium channels. Cold Spring Harbor perspectives in biology 523 3, a003947.

Cull-Candy, S.G., Miledi, R., Trautmann, A., and Uchitel, O.D. (1980). On the release of transmitter at normal, myasthenia gravis and myasthenic syndrome affected human end-plates. J Physiol 299, 621-638.

528

Daniels, R.W., Collins, C.A., Gelfand, M.V., Dant, J., Brooks, E.S., Krantz, D.E., and DiAntonio, A. (2004). Increased expression of the Drosophila vesicular glutamate transporter leads to excess

530 Davis, G.W. (2013). Homeostatic signaling and the stabilization of neural function. Neuron 80, 718531728.

532 Davis, G.W., DiAntonio, A., Petersen, S.A., and Goodman, C.S. (1998). Postsynaptic PKA controls quantal size and reveals a retrograde signal that regulates presynaptic transmitter release in Drosophila. Neuron 20, 305-315.

535 Davis, G.W., and Müller, M. (2015). Homeostatic control of presynaptic neurotransmitter release.

536 Annu Rev Physiol 77, 251-270. 
de Juan-Sanz, J., Holt, G.T., Schreiter, E.R., de Juan, F., Kim, D.S., and Ryan, T.A. (2017). Axonal

538 Endoplasmic Reticulum $\mathrm{Ca}(2+)$ Content Controls Release Probability in CNS Nerve Terminals.

539 Neuron 93, 867-881 e866.

540 Delvendahl, I., and Müller, M. (2019). Homeostatic plasticity-a presynaptic perspective. Curr Opin

$541 \quad$ Neurobiol 54, 155-162.

542 Dockendorff, T.C., Robertson, S.E., Faulkner, D.L., and Jongens, T.A. (2000). Genetic

543 characterization of the 44D-45B region of the Drosophila melanogaster genome based on an F2 lethal

544 screen. Molecular \& general genetics : MGG 263, 137-143.

545 Emptage, N.J., Reid, C.A., and Fine, A. (2001). Calcium stores in hippocampal synaptic boutons mediate short-term plasticity, store-operated Ca2+ entry, and spontaneous transmitter release. Neuron 29, 197-208.

548 Frank, C.A. (2014a). Homeostatic plasticity at the Drosophila neuromuscular junction.

549 Neuropharmacology 78, 63-74.

550 Frank, C.A. (2014b). How voltage-gated calcium channels gate forms of homeostatic synaptic

551 plasticity. Frontiers in cellular neuroscience $8,40$.

552 Frank, C.A., James, T.D., and Muller, M. (2020). Homeostatic control of Drosophila neuromuscular 553 junction function. Synapse 74, e22133.

554 Frank, C.A., Kennedy, M.J., Goold, C.P., Marek, K.W., and Davis, G.W. (2006). Mechanisms 555 underlying the rapid induction and sustained expression of synaptic homeostasis. Neuron 52, 663556677.

557 Frank, C.A., Pielage, J., and Davis, G.W. (2009). A presynaptic homeostatic signaling system

558 composed of the Eph receptor, ephexin, $\mathrm{Cdc} 42$, and $\mathrm{CaV} 2.1$ calcium channels. Neuron 61, 556-569.

559 Gafni, J., Munsch, J.A., Lam, T.H., Catlin, M.C., Costa, L.G., Molinski, T.F., and Pessah, I.N.

560 (1997). Xestospongins: potent membrane permeable blockers of the inositol 1,4,5-trisphosphate 561 receptor. Neuron 19, 723-733.

562 Gaviño, M.A., Ford, K.J., Archila, S., and Davis, G.W. (2015). Homeostatic synaptic depression is

563 achieved through a regulated decrease in presynaptic calcium channel abundance. eLife 4.

564 Genç, Ö., Dickman, D.K., Ma, W., Tong, A., Fetter, R.D., and Davis, G.W. (2017). MCTP is an ER565 resident calcium sensor that stabilizes synaptic transmission and homeostatic plasticity. eLife 6.

566 Goel, P., Dufour Bergeron, D., Bohme, M.A., Nunnelly, L., Lehmann, M., Buser, C., Walter, A.M., 567 Sigrist, S.J., and Dickman, D. (2019). Homeostatic scaling of active zone scaffolds maintains global 568 synaptic strength. J Cell Biol 218, 1706-1724.

569 Gratz, S.J., Goel, P., Bruckner, J.J., Hernandez, R.X., Khateeb, K., Macleod, G.T., Dickman, D., and 570 O'Connor-Giles, K.M. (2019). Endogenous Tagging Reveals Differential Regulation of Ca(2+) 571 Channels at Single Active Zones during Presynaptic Homeostatic Potentiation and Depression. J 572 Neurosci 39, 2416-2429.

573 Handler, A., Graham, T.G.W., Cohn, R., Morantte, I., Siliciano, A.F., Zeng, J., Li, Y., and Ruta, V. 574 (2019). Distinct Dopamine Receptor Pathways Underlie the Temporal Sensitivity of Associative 575 Learning. Cell 178, 60-75 e19.

576 Hazelrigg, T., Levis, R., and Rubin, G.M. (1984). Transformation of white locus DNA in drosophila: 577 dosage compensation, zeste interaction, and position effects. Cell 36, 469-481. 
James, T.D., Zwiefelhofer, D.J., and Frank, C.A. (2019). Maintenance of homeostatic plasticity at the Drosophila neuromuscular synapse requires continuous IP3-directed signaling. eLife 8 . Jeans, A.F., van Heusden, F.C., Al-Mubarak, B., Padamsey, Z., and Emptage, N.J. (2017). Homeostatic Presynaptic Plasticity Is Specifically Regulated by P/Q-type Ca(2+) Channels at

582 Mammalian Hippocampal Synapses. Cell reports 21, 341-350.

583 Kawasaki, F., Collins, S.C., and Ordway, R.W. (2002). Synaptic calcium-channel function in 584 Drosophila: analysis and transformation rescue of temperature-sensitive paralytic and lethal 585 mutations of cacophony. J Neurosci 22, 5856-5864.

Kawasaki, F., Felling, R., and Ordway, R.W. (2000). A temperature-sensitive paralytic mutant defines a primary synaptic calcium channel in Drosophila. J Neurosci 20, 4885-4889.

Kawasaki, F., Zou, B., Xu, X., and Ordway, R.W. (2004). Active zone localization of presynaptic calcium channels encoded by the cacophony locus of Drosophila. J Neurosci 24, 282-285. Genetics 207, 993-1006. inactivation of P/Q-type Ca2+ channels. J Neurosci 20, 6830-6838. C., Millasseau, P., Zeviani, M., et al. (1995). Identification and characterization of a spinal muscular atrophy-determining gene. Cell 80, 155-165. Controls the Presynaptic Inhibition of Neurotransmitter Release. Cell reports 23, 1716-1727.

603 Marder, E., and Goaillard, J.M. (2006). Variability, compensation and homeostasis in neuron and 604 network function. Nat Rev Neurosci 7, 563-574.

605 Marie, B., Pym, E., Bergquist, S., and Davis, G.W. (2010). Synaptic homeostasis is consolidated by 606 the cell fate gene gooseberry, a Drosophila pax3/7 homolog. J Neurosci 30, 8071-8082.

607 Martin, A.R. (1955). A further study of the statistical composition on the end-plate potential. J 608 Physiol 130, 114-122.

609 Meyer, F., and Aberle, H. (2006). At the next stop sign turn right: the metalloprotease Tolloid-related 6101 controls defasciculation of motor axons in Drosophila. Development 133, 4035-4044.

611 Müller, M., and Davis, G.W. (2012). Transsynaptic control of presynaptic $\mathrm{Ca}(2)(+)$ influx achieves 612 homeostatic potentiation of neurotransmitter release. Curr Biol 22, 1102-1108.

613 Newman, Z.L., Hoagland, A., Aghi, K., Worden, K., Levy, S.L., Son, J.H., Lee, L.P., and Isacoff, 614 E.Y. (2017). Input-Specific Plasticity and Homeostasis at the Drosophila Larval Neuromuscular 615 Junction. Neuron 93, 1388-1404 e1310.

616 Oliva, M.K., Perez-Moreno, J.J., O'Shaughnessy, J., Wardill, T.J., and O'Kane, C.J. (2020).

617 Endoplasmic Reticulum Lumenal Indicators in Drosophila Reveal Effects of HSP-Related Mutations 618 on Endoplasmic Reticulum Calcium Dynamics. Front Neurosci 14, 816. 
619 Petersen, S.A., Fetter, R.D., Noordermeer, J.N., Goodman, C.S., and DiAntonio, A. (1997). Genetic

620 analysis of glutamate receptors in Drosophila reveals a retrograde signal regulating presynaptic

621 transmitter release. Neuron 19, 1237-1248.

622 Pozo, K., and Goda, Y. (2010). Unraveling mechanisms of homeostatic synaptic plasticity. Neuron

$62366,337-351$.

624 Raimer, A.C., Singh, S.S., Edula, M.R., Paris-Davila, T., Vandadi, V., Spring, A.M., and Matera, 625 A.G. (2020). Temperature-sensitive spinal muscular atrophy-causing point mutations lead to SMN 626 instability, locomotor defects and premature lethality in Drosophila. Dis Model Mech 13.

627 Sen, A., Yokokura, T., Kankel, M.W., Dimlich, D.N., Manent, J., Sanyal, S., and Artavanis-

628 Tsakonas, S. (2011). Modeling spinal muscular atrophy in Drosophila links Smn to FGF signaling. J

629 Cell Biol 192, 481-495.

630 Simkus, C.R., and Stricker, C. (2002). The contribution of intracellular calcium stores to mEPSCs 631 recorded in layer II neurones of rat barrel cortex. J Physiol 545, 521-535.

632 Smith, L.A., Peixoto, A.A., Kramer, E.M., Villella, A., and Hall, J.C. (1998). Courtship and visual 633 defects of cacophony mutants reveal functional complexity of a calcium-channel alpha1 subunit in 634 Drosophila. Genetics 149, 1407-1426.

635 Spring, A.M., Brusich, D.J., and Frank, C.A. (2016). C-terminal Src Kinase Gates Homeostatic 636 Synaptic Plasticity and Regulates Fasciclin II Expression at the Drosophila Neuromuscular Junction. 637 PLoS genetics 12, e1005886.

638 Spring, A.M., Raimer, A.C., Hamilton, C.D., Schillinger, M.J., and Matera, A.G. (2019).

639 Comprehensive Modeling of Spinal Muscular Atrophy in Drosophila melanogaster. Frontiers in 640 molecular neuroscience 12, 113.

641 Summerville, J.B., Faust, J.F., Fan, E., Pendin, D., Daga, A., Formella, J., Stern, M., and McNew, 642 J.A. (2016). The effects of ER morphology on synaptic structure and function in Drosophila 643 melanogaster. Journal of cell science 129, 1635-1648.

644 Turrigiano, G.G. (2008). The self-tuning neuron: synaptic scaling of excitatory synapses. Cell 135, $645422-435$.

646 Vazquez-Martinez, O., Canedo-Merino, R., Diaz-Munoz, M., and Riesgo-Escovar, J.R. (2003).

647 Biochemical characterization, distribution and phylogenetic analysis of Drosophila melanogaster

648 ryanodine and IP3 receptors, and thapsigargin-sensitive Ca2+ ATPase. Journal of cell science 116, 649 2483-2494.

650 Venkatesh, K., and Hasan, G. (1997). Disruption of the IP3 receptor gene of Drosophila affects larval 651 metamorphosis and ecdysone release. Curr Biol 7, 500-509.

652 Wagh, D.A., Rasse, T.M., Asan, E., Hofbauer, A., Schwenkert, I., Durrbeck, H., Buchner, S., 653 Dabauvalle, M.C., Schmidt, M., Qin, G., et al. (2006). Bruchpilot, a protein with homology to 654 ELKS/CAST, is required for structural integrity and function of synaptic active zones in Drosophila. 655 Neuron 49, 833-844.

656 Wang, X., Pinter, M.J., and Rich, M.M. (2016). Reversible Recruitment of a Homeostatic Reserve 657 Pool of Synaptic Vesicles Underlies Rapid Homeostatic Plasticity of Quantal Content. J Neurosci 36, 658 828-836.

659 Wilcox, R.A., Primrose, W.U., Nahorski, S.R., and Challiss, R.A. (1998). New developments in the 660 molecular pharmacology of the myo-inositol 1,4,5-trisphosphate receptor. Trends Pharmacol Sci 19 , 661 467-475. 
Yeates, C.J., and Frank, C.A. (2020). A class of synaptic signaling molecules required for homeostatic potentiation also tunes homeostatic depression. bioRxiv, 2020.2010.2012.336883. Yeates, C.J., Zwiefelhofer, D.J., and Frank, C.A. (2017). The Maintenance of Synaptic Homeostasis at the Drosophila Neuromuscular Junction Is Reversible and Sensitive to High Temperature. eNeuro 4 . presynaptic calcium influx. J Neurosci 31, 7492-7496. receptor $\mathrm{Ca} 2+$ release channels. Molecular mechanism and isoform selectivity. J Biol Chem 276, $13810-13816$. of OK371-Gal4 and $\boldsymbol{U A S}$-VGlut. (A) NMJ electrophysiological data for miniature excitatory postsynaptic potentials (mEPSP), excitatory postsynaptic potentials (EPSP), and quantal content (QC). Data are normalized to WT $\left(w^{1118}\right)$ values. VGlut, OK371/+ NMJs have increased mEPSP but normal EPSP because of decreased QC, indicative of successful PHD. (*** $p<0.001$ vs. WT by one-way ANOVA with Tukey's post-hoc). (B) Representative electrophysiological traces. Large traces are EPSPs; small traces are mEPSPs. Scale bars for EPSPs (mEPSPs) are $5 \mathrm{mV}(1 \mathrm{mV})$ and $50 \mathrm{~ms}(1000$ ms). (C) Raw data for mEPSPs. (D) Raw data for EPSPs. (E) Raw data for QC. For (C)-(E), bars are averages and error bars are \pm SEM. $* * * p<0.001$ vs. WT or vs. $O K 371 /+$; $p<0.05$ vs. OK371/+; analyses by one-way ANOVA with Tukey's post-hoc.

Figure 2. An electrophysiology screen in a PHD-challenged genetic background. (A) Crossing scheme for generating larvae for electrophysiological recording. Each animal recorded had a homeostatic challenge provided by VGlut overexpression and a concurrent heterozygous or RNAi condition. (B) Data distribution for screened conditions (x-axis = average EPSP for condition; y-axis $=$ average QC for condition). Green =UAS-VGlut, OK371-Gal4/+. Red =UAS-VGlut, OK371Gal4/RyRE4340K; itpr90B/+. Purple = UAS-VGlut, OK371-Gal4/+; UAS-Smn[RNAi] $]^{J F 02057 /+.}$. Dotted line: EPSP value two standard deviations below UAS-VGlut, OK371-Gal4/+ chosen as a cut off for potential follow-up hits. (C) Average EPSPs for screened conditions. All conditions have a $U A S$-VGlut, OK371-Gal4/+ genetic background. ">" denotes as UAS construct or RNAi line being driven in motor neurons by OK371-Gal4. "+" denotes additional mutations present as heterozygotes. Top dotted line denotes $U A S-V G l u t$, OK371-Gal4/+ average. Bottom dotted line denotes two standard deviations below UAS-VGlut, OK371-Gal4/+ average.

Figure 3. Double heterozygous loss of the itpr and $\boldsymbol{R} \boldsymbol{H} R$ genes interacts with the PHD challenge to diminish neurotransmission. Note: Traces and data for $O K 371 /+$ and $V G l u t, O K 371 /+$ are repeated from Figure 1, for genetic background comparison. Abbreviations are as in Figure 1. (A) NMJ electrophysiological data for mEPSP, EPSP, and QC. Data are normalized to $O K 371 /+$ values. ${ }^{*} p<$ $0.05, * * p<0.01$, and $* * * p<0.001$ vs. OK371/+; \#\# $p<0.01$ and \#\#\# $p<0.001$ vs. OK371/RyRE4340K; itpr90B/+; analyses by one-way ANOVA with Tukey's post-hoc. (B) Raw data for mEPSPs. (C) Raw data for EPSPs. (D) Raw data for QC. For (B)-(D), bars are averages and error bars are \pm SEM. $* p<0.05, * * p<0.01$, and $* * * p<0.001$ by one-way ANOVA across genotypes, 
706

707

708

709

710

711

712

713

714

715

716

717

718

719

720

721

722

723

724

725

726

727

728

729

730

731

732

733

734

735

736

737

738

739

740

741

742

743

744

745

746

747

748

749

750

751

752

753 with Tukey's post-hoc. (E) Representative electrophysiological traces with standard deviation (S.D.), coefficient of variation (C.V.) and range values for EPSPs. The S.D., C.V., and range were significantly higher for VGlut, OK371/RyR $R^{E 4340 K}$; itpr ${ }^{90 B} /+$ vs. its genetic control, OK371/RyR $R^{E 4340 K}$; itpr $r^{90 B} /+$. * $p$ $<0.05, * * p<0.01$ by one-way ANOVA across genotypes, with Tukey's post-hoc. Scale bars as in Figure 1 .

Figure 4. No discernible NMJ growth defects. NMJs of third instar larvae (same genotypes as Figure 3) were analysed by immunostaining, co-staining with anti-DLG for the postsynaptic density and antiBrp to check for apposed presynaptic active zones. (A) DLG boutons counted for Segment A2, NMJ 6/7. (B) DLG boutons counted for Segment A3, NMJ 6/7. No significant differences were found across genotypes ( $p>0.9$ for every possible head-to-head comparison, one-way ANOVA).

Figure 5. Genetic impairment of itpr combined with pharmacological impairment of RyR phenocopies prior genetic findings. Note: Untreated data for $O K 371 /+$ and $V G l u t, O K 371 /+$ are repeated from Figure 1, for genetic background comparison. (A) Raw data for mEPSPs (left); raw data for EPSPs (middle); raw data for QC (right) for untreated genotypes as shown; bars are averages and error bars are \pm SEM. (B) Data as in (A) but with $25 \mu \mathrm{M}$ Dantrolene added to NMJ preps. * $p<0.05$, $* * p<0.01$, and $* * * p<0.001$ by one-way ANOVA with Tukey's post-hoc. (C) Representative EPSP traces. Scale bars are as in Figure 1.

Figure 6. Additional pharmaco-genetic combinations phenocopy the genetic conditions. (A) Raw data for mEPSPs (left); raw data for EPSPs (middle); raw data for QC (right) for untreated genotypes as shown; bars are averages and error bars are \pm SEM. (B) Data as in (A) but with $25 \mu \mathrm{M}$ Dantrolene added to NMJ preps (C) Data as in (A) but with $20 \mu \mathrm{M}$ Xestospongin C added to NMJ preps. (D) Representative EPSP traces. Scale bars are as in Figure 1. ${ }^{*} p<0.05, * * p<0.01$, and $* * * p<0.001$ by Student's T-Test comparing a control dataset (no VGlut overexpression) vs. an experimental dataset (VGlut overexpression).

Figure 7. $\mathrm{Ca}^{2+}$ concentration-sensitivity of PHD execution. (A) Log-log plots of recording saline $\left[\mathrm{Ca}^{2+}\right]$ vs. QC corrected for non-linear summation for WT, OK371/+, and VGlut, OK371/+ conditions. Across the range of $\left[\mathrm{Ca}^{2+}\right]$ examined, there is no significant difference in calcium cooperativity for these conditions (Nonlinear Regression, $p=0.91$ ). (B) Data plotted as in (A) but this time with a double heterozygous $R y R^{E 4340 K /+}$; itpr $r^{90 B} /+$ genetic background. Across the range of $\left[\mathrm{Ca}^{2+}\right]$ examined, there is no significant difference in calcium cooperativity for these conditions (Nonlinear Regression, $p=$ 0.78). (C) Raw data for mEPSPs (left); raw data for EPSPs (middle); raw data for QC (right). All data are for the indicated NMJ genotypes in $0.2 \mathrm{mM}\left[\mathrm{Ca}^{2+}\right.$; bars are averages and error bars are $\pm \mathrm{SEM}$. For mEPSPs, ${ }^{*} p<0.05$ and $* * * p<0.001$ by Student's T-Test, comparing PHD-challenged genotypes vs. unchallenged genetic controls. For EPSPs and QC, ${ }^{*} p<0.05$, $* * p<0.01$, and $* * * p<0.001$ vs. OK371/+; \#\# $p<0.01$; EPSP and QC analyses done across multiple genotypes by one-way ANOVA with Tukey's post-hoc.

Figure 8. Partial impairment of Cav2/Cacophony and PHD. (A) Crossing scheme for generating larvae for electrophysiological recording. Male larvae were hemizygous for the $c a c^{S}$ hypomorphic mutation. (B) Raw data for mEPSPs. (C) Raw data for EPSPs. (D) Raw data for QC. For (B)-(D), bars are averages and error bars are \pm SEM. $* p<0.05, * * p<0.01$, and $* * * p<0.001$ by Student's T-Test comparing the control $\mathrm{cac}^{S}$ dataset (no VGlut overexpression) vs. the experimental $c a c^{S}$ dataset (VGlut overexpression). 
Figure 9. Model for how multiple calcium sources interact with the process of PHD. Under baseline conditions, Cav2-type calcium channels contribute to synapse function, as may RyRs and $\mathrm{IP}_{3}$ Rs. Under conditions inducing PHD, synaptic vesicles are enlarged, and QC is decreased, through regulation of sources of calcium. When PHD challenge is coupled with concomitant impairment of RyR and $\mathrm{IP}_{3} \mathrm{R}$ channels, evoked potentials are significantly diminished.

\section{$7 \quad$ Conflict of Interest}

761

762

763

764

765

766

767

768

769

770

771

772

773

774

775

776

777

778

779

780

781

782

783

The authors declare that the research was conducted in the absence of any commercial or financial relationships that could be construed as a potential conflict of interest.

\section{Author Contributions}

C.J.Y. and C.A.F. both did the following: designed research, performed research, analysed data, and wrote and edited the paper.

\section{$9 \quad$ Funding}

Funding supporting this work includes an NSF Grant (1557792) and an NIH/NINDS Grant (R01NS085164) to C.A.F. C.J.Y. was supported in part by an NIH/NINDS Predoctoral Training Grant to the University of Iowa (UI) Interdisciplinary Graduate Program in Neuroscience (T32NS007421 - PI Daniel T. Tranel), as well as a post-comprehensive exam predoctoral summer fellowship and a Ballard and Seashore Dissertation fellowship via the Graduate College at UI.

\section{Acknowledgements}

We thank members of the Frank lab for helpful comments. We thank the laboratories of Drs. Tina Tootle, Fang Lin, Toshihiro Kitamoto, Pamela Geyer, and Lori Wallrath for helpful discussions. We also thank Drs. Toshihiro Kitamoto, Joshua Weiner, Christopher Stipp, and Mark Stamnes for helpful feedback on an earlier written version of this study. An earlier version of this manuscript was released as a pre-print at bioRxiv, (Yeates and Frank, 2020).

\section{Supplementary Material}

Please see Supplementary Table S1 for raw data from the electrophysiology screen and a legend explaining the table.

\section{Data Availability Statement}

The raw data supporting the conclusions in this article will be made available by the authors, without undue reservation. 
A

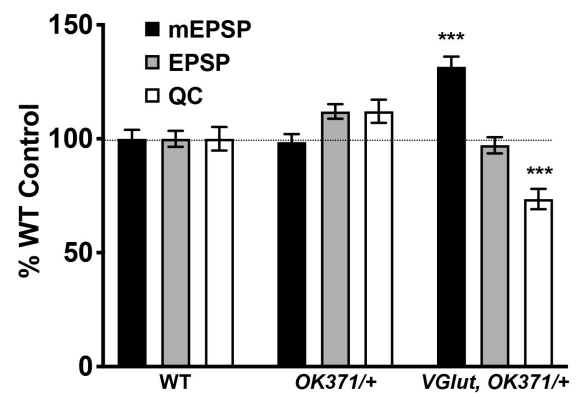

B
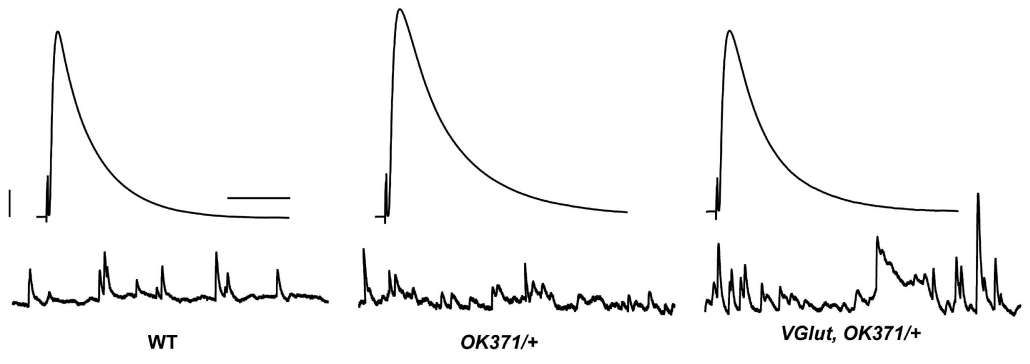

E

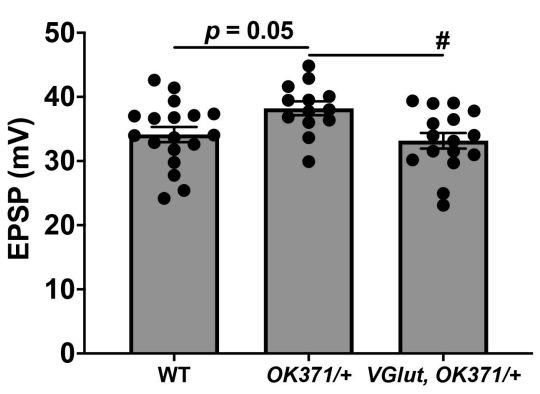

D

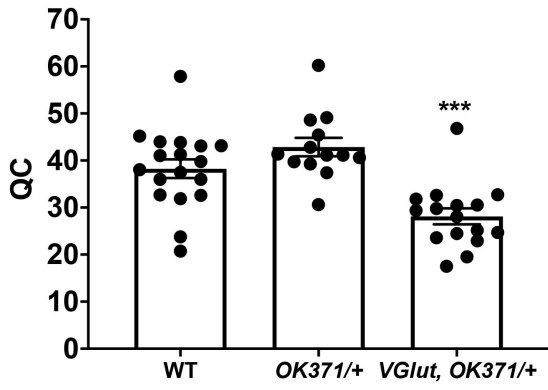

C

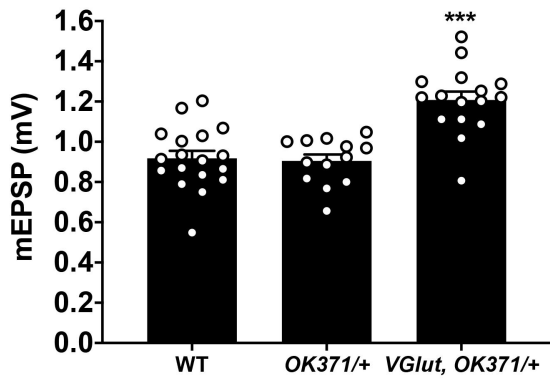




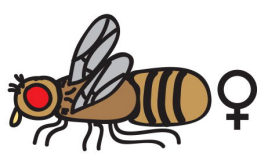

w; UAS-VGlut, OK371-Gal4 X mut OR UAS-gene[RNAi]

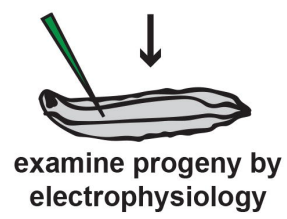

C

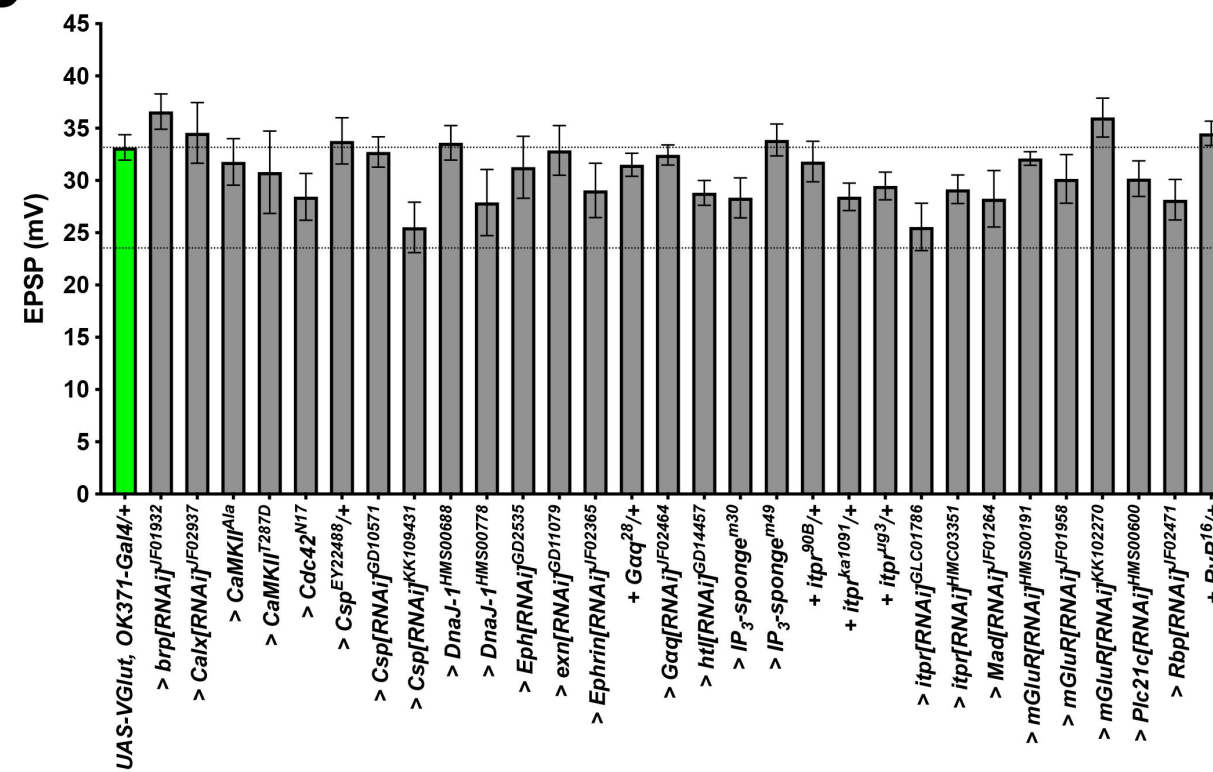

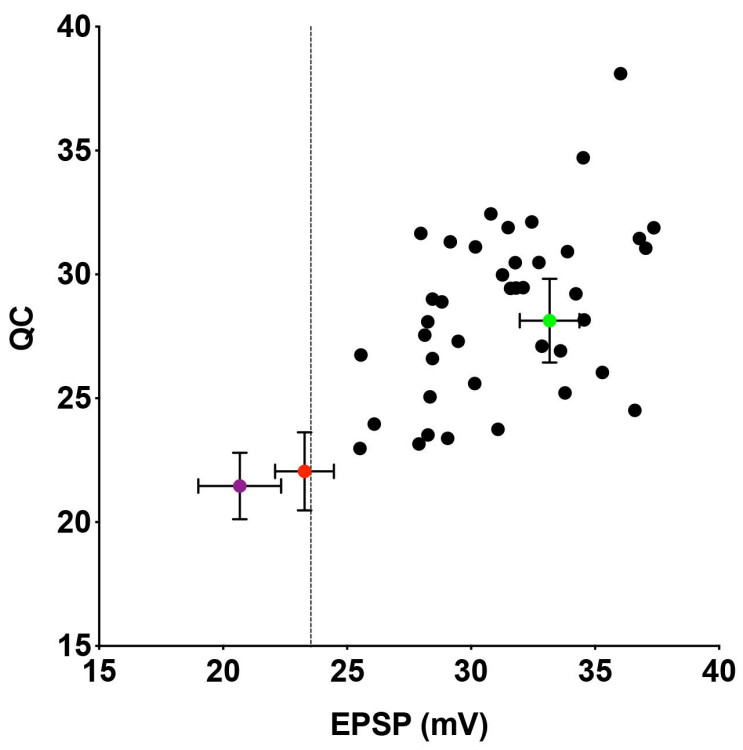


A

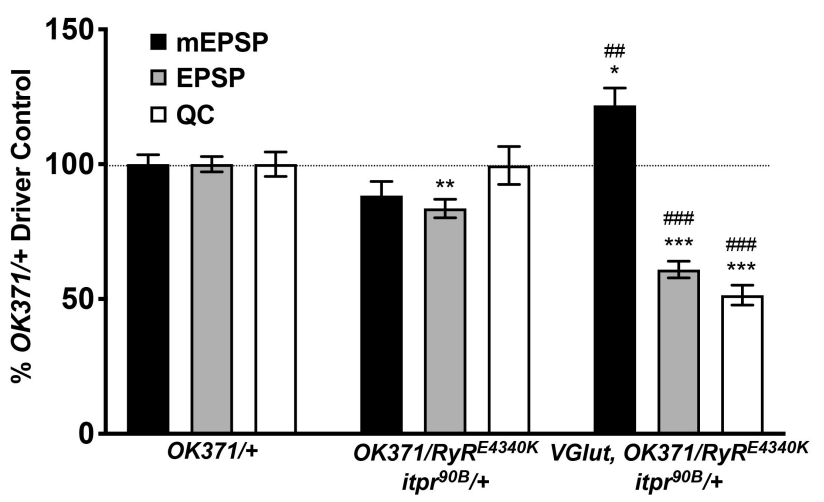

C

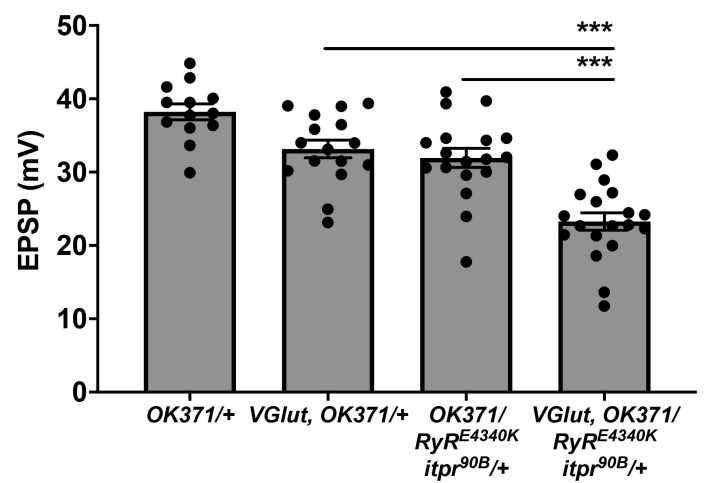

E

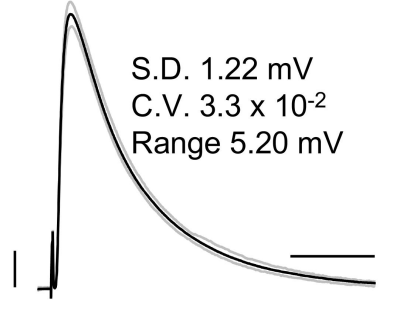

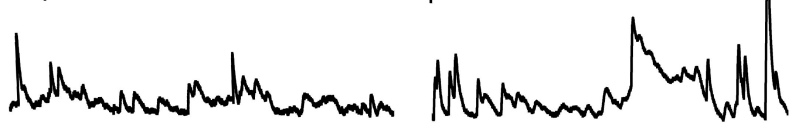
OK371/+

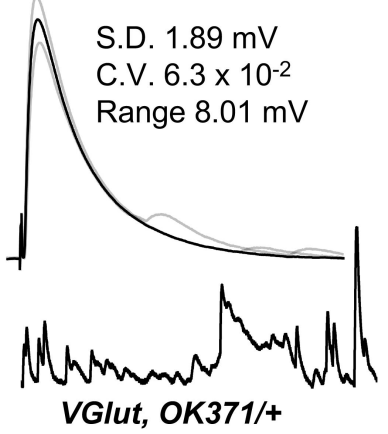

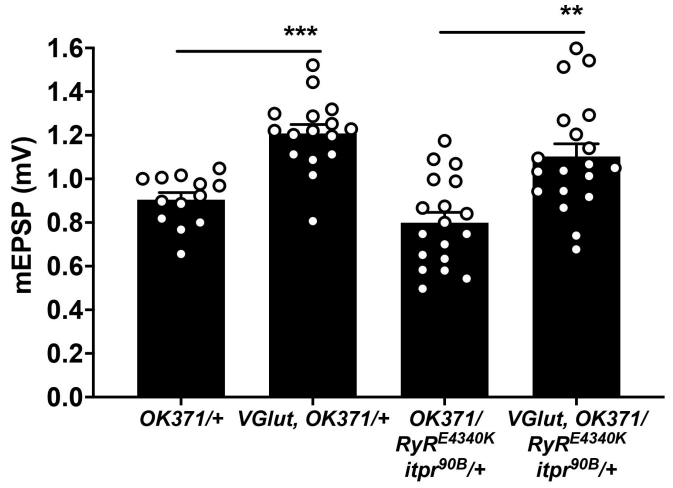

D

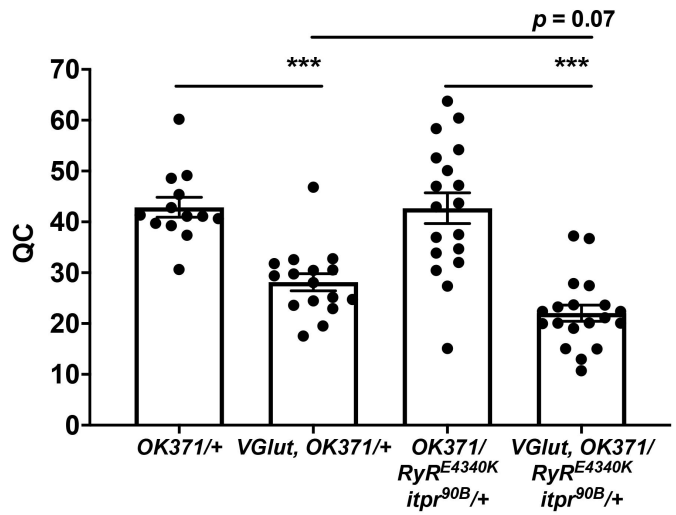

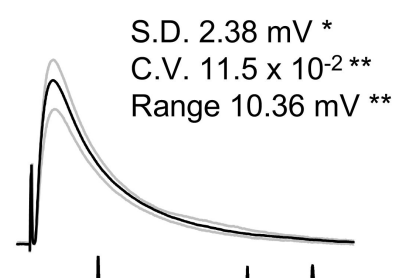

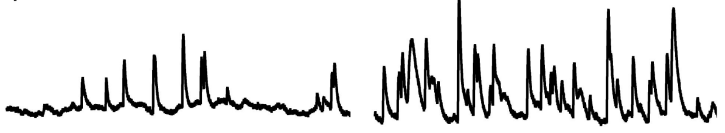
OK371/RyR ${ }^{E 4340 K}$ itpr ${ }^{90 \mathrm{~B} /+}$ 
A

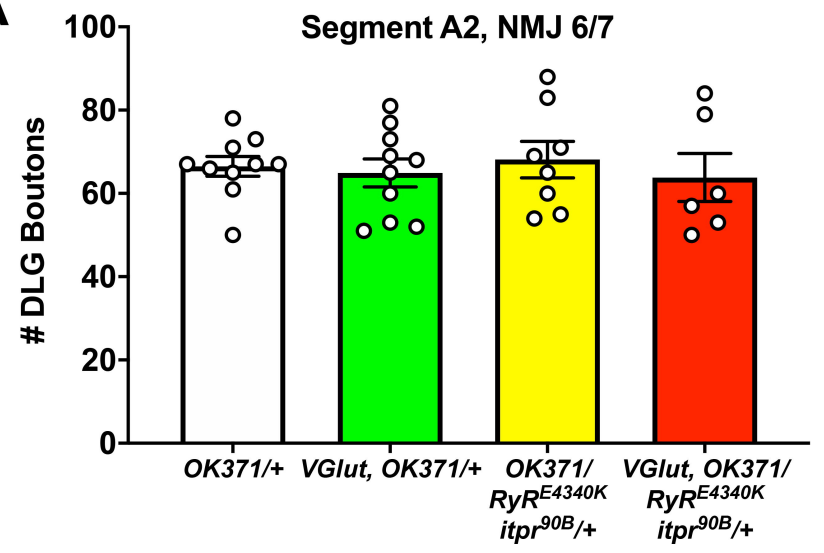

B
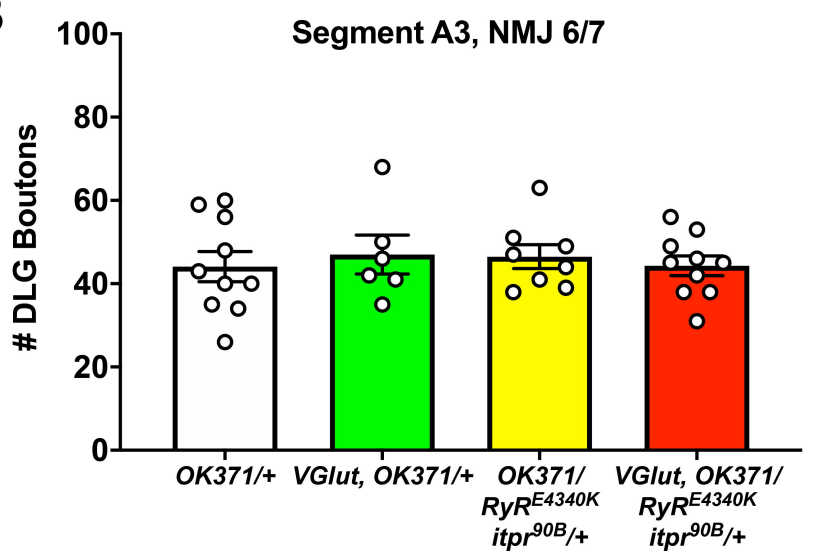

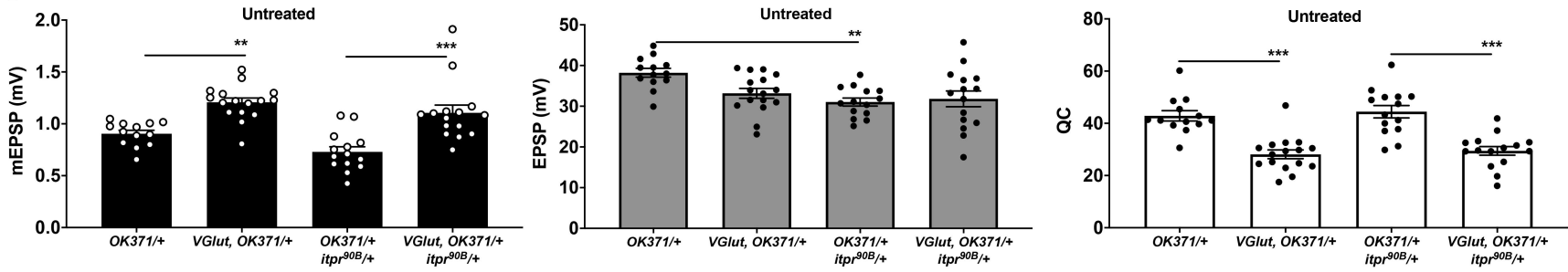

B
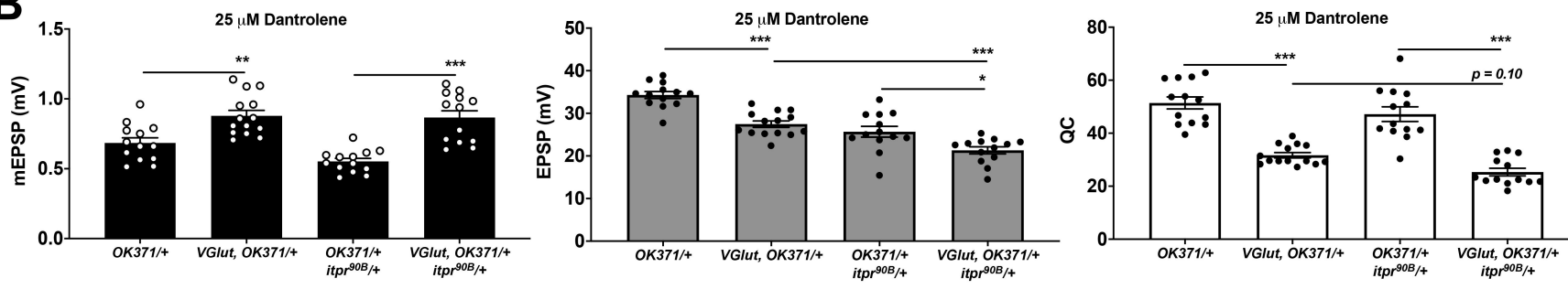

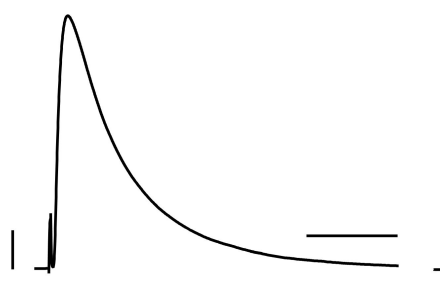

OK371/+

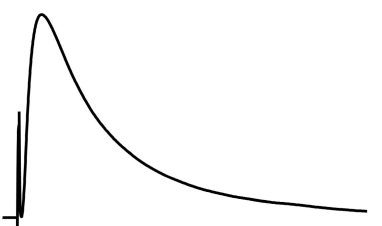

VGlut, OK371/+

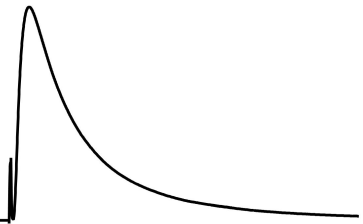

OK371/+ itpr $^{90 \mathrm{~B} /+}$

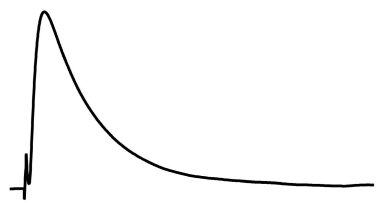

VGlut, OK371/+ itpr ${ }^{908 /+}$ 
A

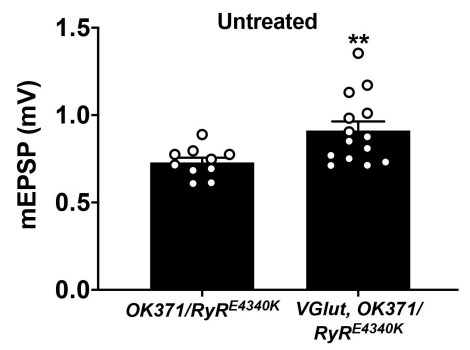

B

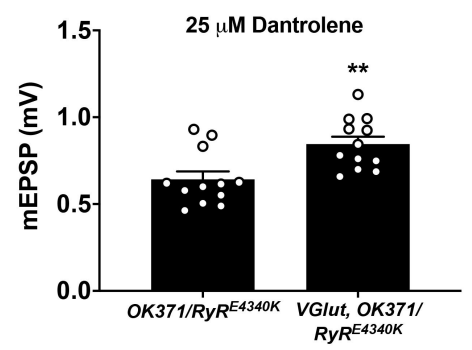

C

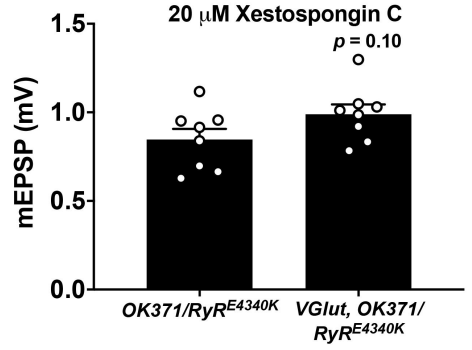

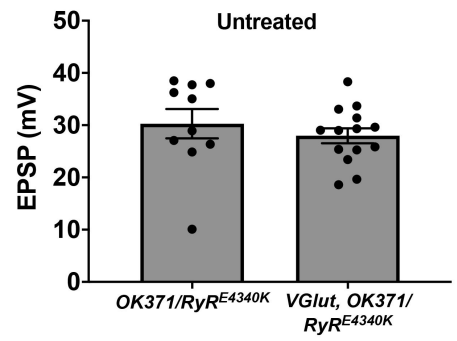
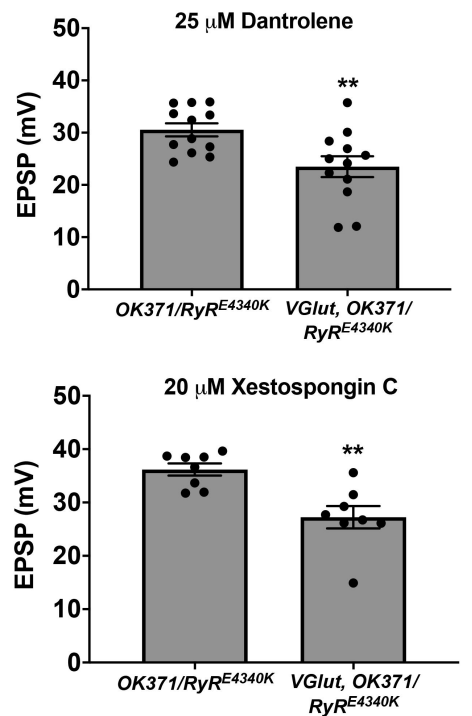
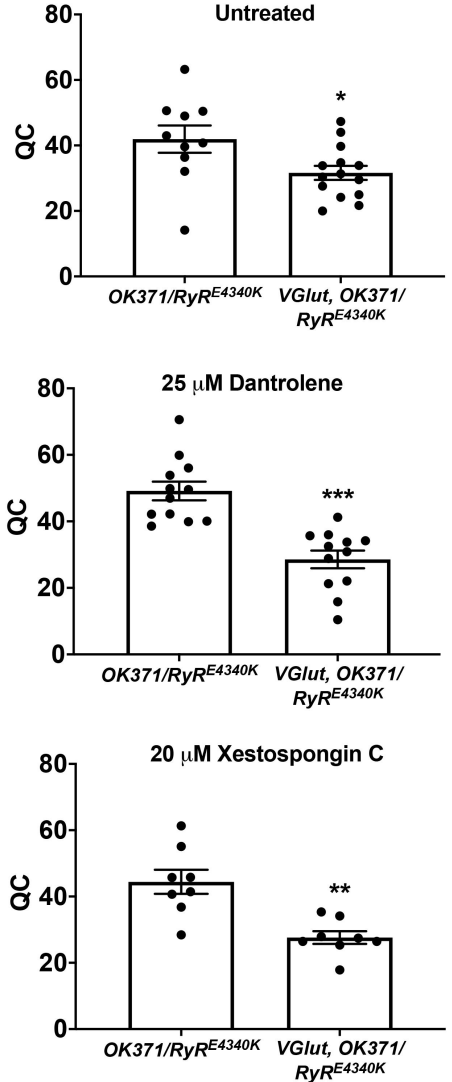

D

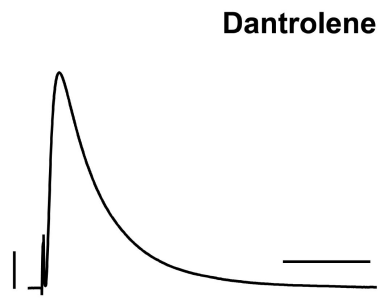

OK371/RyR $R^{E 4340 K}$

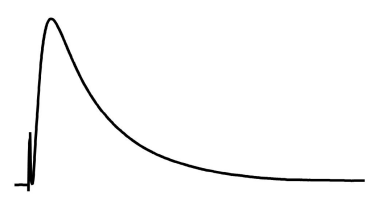

VGlut, OK371/RyR E4340K $^{2}$

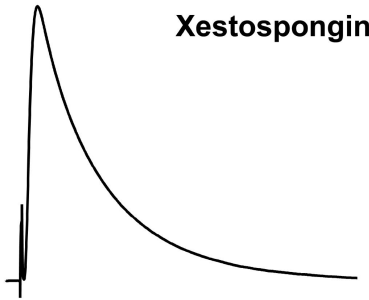

OK371/RyR $R^{E 4340 K}$

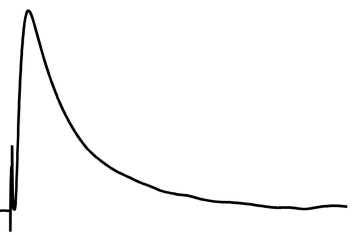

VGlut, OK371/ RyR $R^{E 4340 K}$ 
A

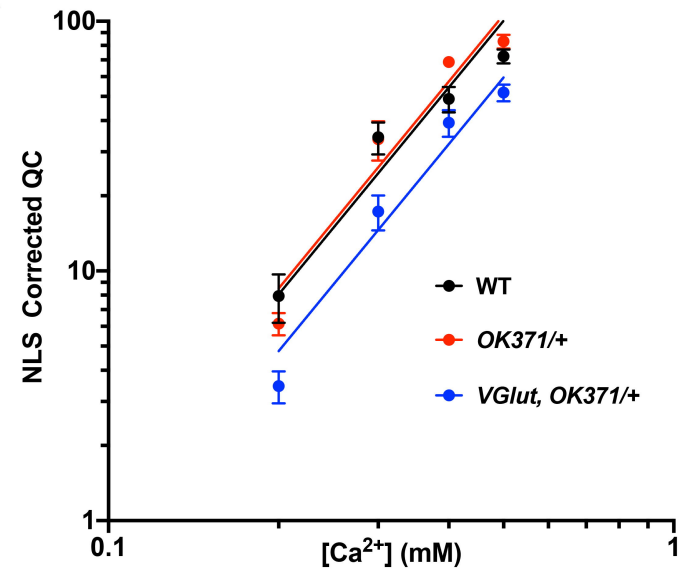

B

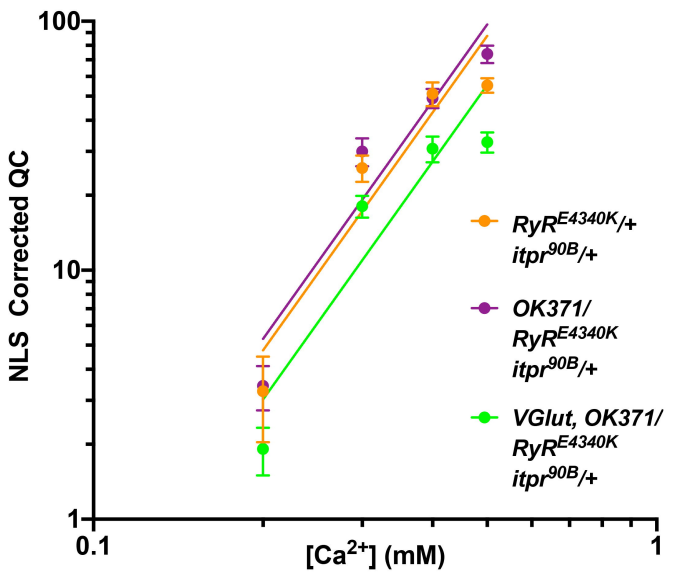

C

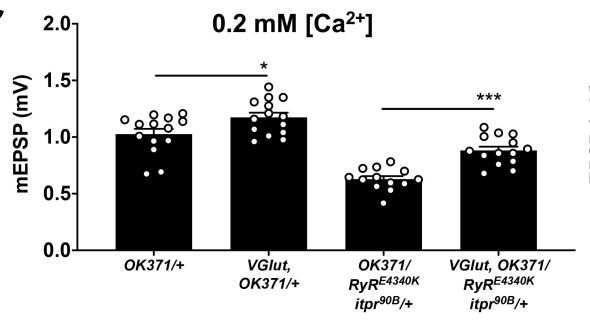

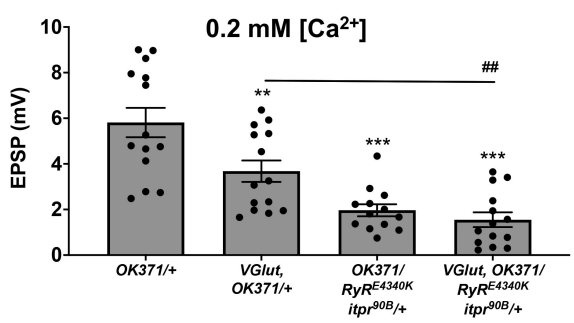

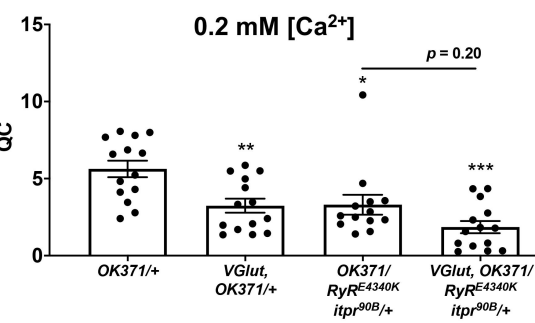




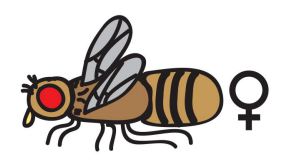

$\operatorname{cac}^{s} / \mathrm{cac}^{s}$

X w/Y; UAS-VG/ut, OK371-Gal4/(Bal)

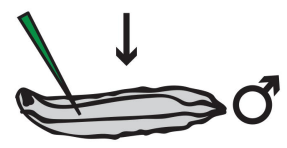

$\operatorname{cac}^{S}$ YY; UAS-VGlut, OK371-Gal4/+

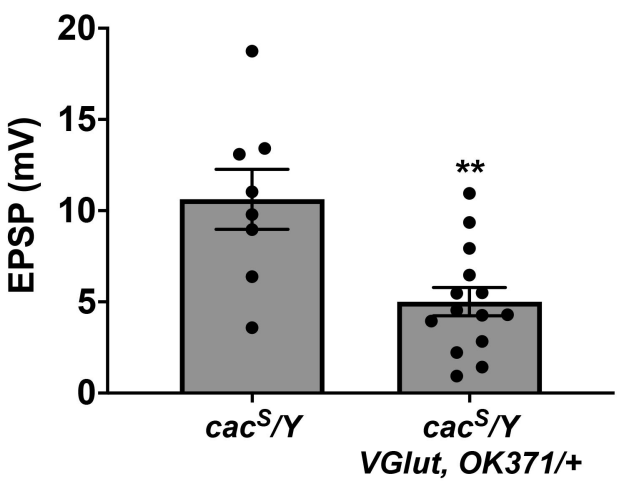

B

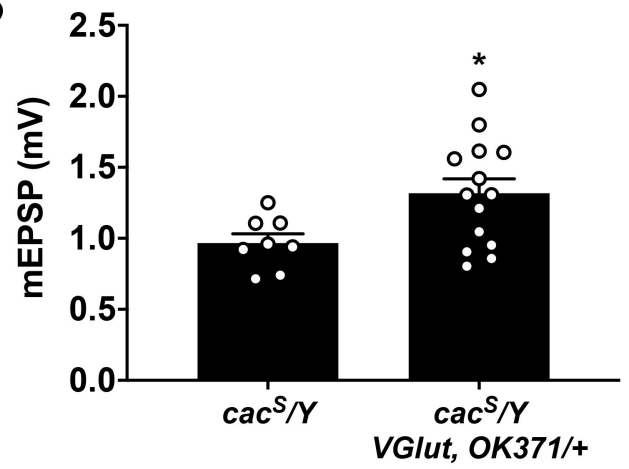

D

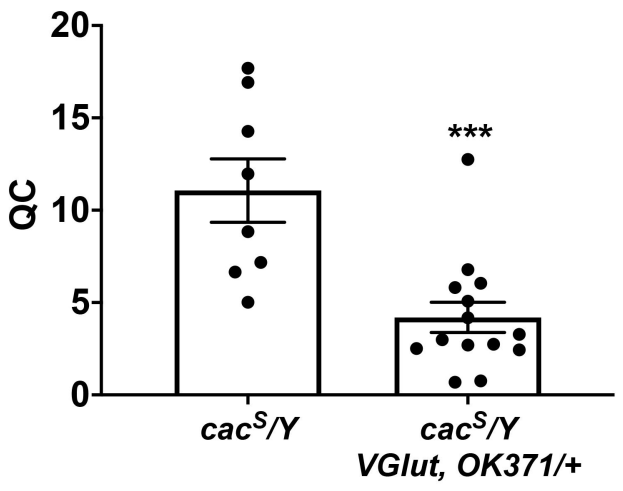


WT

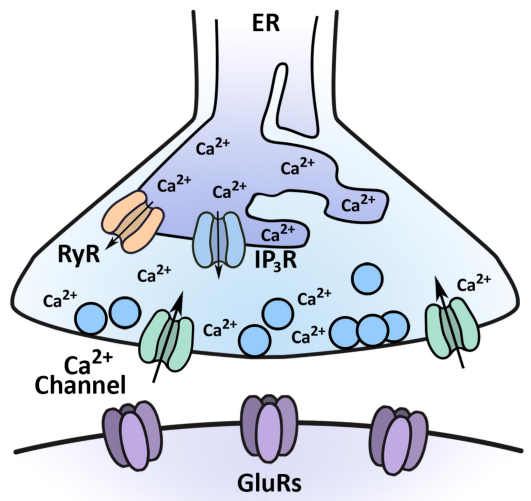

Evoked potentials normal
Homeostatic Depression

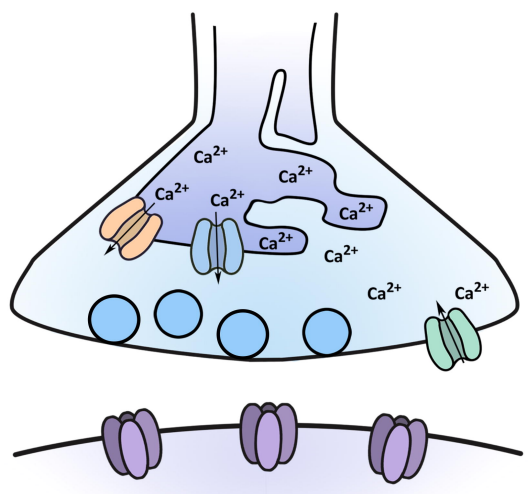

Evoked potentials $\approx$ normal
Homeostatic Depression

+ RyR itpr Impairment

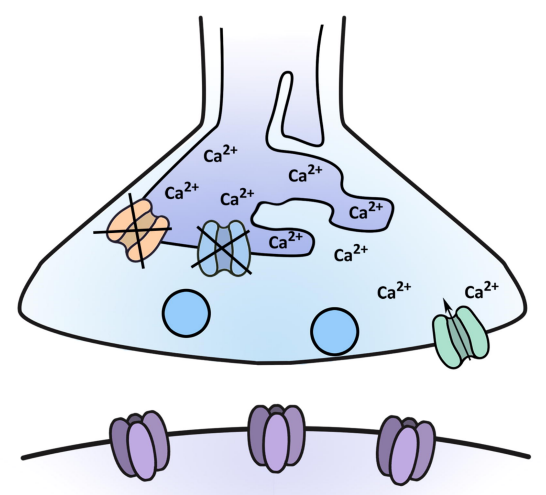

Evoked potentials small 